\title{
On nature of the scalar-isoscalar mesons in the uniformizing-variable method based on analyticity and unitarity
}

\author{
Yurii S. Surovtsev ${ }^{1}$, Petr Bydžovský ${ }^{2}$, Valery E. Lyubovitskij ${ }^{3 *}$ \\ 1 Bogoliubov Laboratory of Theoretical Physics, \\ Joint Institute for Nuclear Research, \\ 141980 Dubna, Russia \\ 2 Nuclear Physics Institute, Czech Academy of Sciences, \\ Řež near Prague 25068, Czech Republic \\ 3 Institut für Theoretische Physik, Universität Tübingen, \\ Kepler Center for Astro and Particle Physics, \\ Auf der Morgenstelle 14, D-72076 Tübingen, Germany
}

(Dated: November 6, 2018)

\begin{abstract}
The experimental data on the processes $\pi \pi \rightarrow \pi \pi, K \bar{K}, \eta \eta, \eta \eta^{\prime}$ in the $I^{G} J^{P C}=0^{+} 0^{++}$channel have been jointly analyzed to study the status and nature of the $f_{0}$. The method of analysis is based on analyticity and unitarity and uses an uniformization procedure. Some spectroscopic implications from results of the analysis are discussed.
\end{abstract}

PACS numbers: 11.55.Bq,11.80.Gw,12.39.Mk,14.40.Cs

Keywords: coupled-channel formalism, meson-meson scattering, scalar and pseudoscalar mesons

\section{INTRODUCTION}

The problem of interpretation of scalar mesons is tightly related to the most profound topics in particle physics which concern the QCD vacuum (see, e.g., the review "Note on scalar mesons" in [1]). These mesons are expected to be composed of the $q \bar{q}$ or the lightest 4-quark states, meson-meson molecules or gluonium states. It is disconcerning that up to now a description of this mesonic sector is far from being complete despite of the big effort devoted to studying various aspects of the problem (for recent reviews see, e.g. 2- 5]). Parameters of the scalar mesons, their nature and status of some of them is still not settled [1]. Especially, this concerns the $f_{0}(600) / \sigma$ meson and $K_{0}^{*}(900) / \kappa(800)$ meson. For example, the mass of the former obtained in the Breit-Wigner or $K$-matrix approaches ranges in various analyses in the interval of about 400-1200 MeV [1]. According to the prediction by Weinberg [6] based on the mended symmetry the mass of the $\sigma$ should be near the mass of the $\rho$-meson. As to the mass of the lowest scalar glueball, various non-perturbative QCD methods give also very different results. From the QCD sum rules 7] one has found a scalar-isoscalar meson of the gluonium nature with a mass about $1000 \mathrm{MeV}$ and with the $\pi \pi$-decay width about $500 \mathrm{MeV}$. This is in agreement with the recent unquenched-lattice simulation using dynamical fermions [8] but it diverges from recent calculations on the quenched anisotropic lattices of the glueball spectrum where the mass of the lowest glueball is about $1710 \mathrm{MeV}[\underline{9}$.

The width of the $f_{0}(600)$ (in various experiments and analyses) also has a large spread 600-1000 MeV according to an estimate of the Particle Data Group team [1]. Note also the works in which one obtained a very small value of $35 \pm 12 \mathrm{MeV}$ [10] and the very large one of about $3200 \mathrm{MeV}$ [11]. The prediction for the $\sigma$-meson width on the basis of saturating the superconvergence dispersive sum rules is larger than about $670 \mathrm{MeV}$ [12]. The theoretical conclusions about widths of glueballs, especially about the lightest one, are also very different in various approaches. In Ref. [13] the authors used an effective QCD Lagrangian with the broken scale and chiral symmetry, where a glueball is introduced to theory as a dilaton and its existence is related to breaking of scale symmetry in QCD. Then the $\pi \pi$ decay width of the glueball, estimated using low-energy theorems, is $\Gamma(G \rightarrow \pi \pi) \approx 0.6 \mathrm{GeV} \times\left(m_{G} / 1 \mathrm{GeV}\right)^{5}$, where $m_{G}$ is the glueball mass. I.e., for the glueball with the mass about $1 \mathrm{GeV}$ (if it exists), the width is near $600 \mathrm{MeV}$. Though a use of the above formula is doubtful above $1 \mathrm{GeV}$, a tendency for the glueball to be wide is apparently seen. This is supported by arguments given in 14] that the glueball width is larger than the ones of the surrounding $q \bar{q}$ states. On the other hand, in Ref. [15], where the two-pseudoscalar and two-photon decays of the scalars between 1-2 GeV were

* On leave of absence from the Department of Physics, Tomsk State University, 634050 Tomsk, Russia 
analyzed in the framework of a chiral Lagrangian and the glueball was included as a flavor-blind composite mesonic field, the glueball was found to be rather narrow in accordance with the former findings of Ref. [16].

Up to now the nature of the $f_{0}(980)$ is not clearly resolved. Besides a $q \bar{q}[17,18,20,21$, subject to serious criticism, there are recent arguments for a 4-quark state (as the $a_{0}(980)$ ) [19], a $K \bar{K}$ molecule [22 24] and a $\eta \eta$ bound state 25 27].

Existence of the $f_{0}(1370)$ meson is still not obvious. In some works, e.g., in [28, 29] one did not find any evidence for the existence of the $f_{0}(1370)$. In Ref. [30] also the best description of $\pi \pi \rightarrow \pi \pi, K \bar{K}$ was obtained without the $f_{0}(1370)$, and it was shown that the $K \bar{K}$ scattering length is very sensitive to whether this state exists or not. On the other hand, in Ref. [31] a number of data requiring apparently the existence of the $f_{0}(1370)$ is indicated: the Crystal Barrel data on $\bar{p} p \rightarrow \eta \eta \pi^{0}$ [32] and on $\bar{p} p \rightarrow 3 \pi^{0}$ [33], the BES data on $J / \psi \rightarrow \phi \pi^{+} \pi^{-}$[34]; the $f_{0}$ (1370) appears also in the GAMS data for $\pi^{+} \pi^{-} \rightarrow \pi^{0} \pi^{0}$ at large $|t|$ [35]. For example, in [36] it was shown within the so-called "hidden gauge formalism" that the $f_{0}(1370)$ might be dynamically generated from the $\rho \rho$ interaction.

Especially it is worth to discuss the situation with scalar states in the $1500 \mathrm{MeV}$ region. First, a state, observed in this region could be a real candidate for the lightest glueball (see, e.g., Ref. [16]). In the model-independent analyses of data on the processes $\pi \pi \rightarrow \pi \pi K \bar{K}, \eta \eta, \eta \eta^{\prime}$ using different uniformizing variables [25 27, 30, 37 40], a wide state $f_{0}(1500)$ was obtained whereas in many other works, which analyzed mainly the production and decay of mesons, as cited in the PDG tables [1], the rather narrow $f_{0}(1500)$ is obtained. Therefore, we have supposed [30, 38] that the wide $f_{0}(1500)$, observed in the multi-channel $\pi \pi$ scattering, indeed, is a superposition of two states, narrow $(q \bar{q})$ and broad (glueball). The former is just observed in the processes of decay and production of mesons. An indication about nature of the latter follows from the fact that the $f_{0}(1500)$ is coupled with the approximately equal strength with the $\pi \pi, K \bar{K}$ and $\eta \eta$ systems [25, 26, 30, 37 39] and from the arguments of Ref. [14] on the glueball width. These suppositions are in some accordance with the results of the combined K-matrix analysis [41] of the GAMS data on $\pi^{-} p \rightarrow \pi^{0} \pi^{0} n, \eta \eta n, \eta \eta^{\prime} n$ [42], BNL data on $\pi^{-} p \rightarrow K \bar{K} n$ [43] and Crystal Barrel data on $p \bar{p}($ at rest $) \rightarrow \pi^{0} \pi^{0} \pi^{0}, \pi^{0} \pi^{0} \eta, \pi^{0} \eta \eta$ [44, 45], which say that in the $1500 \mathrm{MeV}$ region there are the narrow $f_{0}(1500)$ and very wide $f_{0}\left(1530_{-250}^{+90}\right)$.

The $f_{0}(1710)$ has most likely the dominant $s \bar{s}$ component (see, e.g., Refs. [30, 46] and the review "Note on scalar mesons" in [1]). Note, however, that the QCD sum rules [47] and the $K$-matrix method [48] showed that both $f_{0}(1500)$ and $f_{0}(1710)$ are mixed states with a large admixture of the glueball component. There are also schemes [28, 49] in which the coupling of two gluons (of a scalar glueball) with $n \bar{n}$ ( $n$ is nonstrange $u$ or $d$ quark) appears to be suppressed by chiral symmetry [50] increasing the relative contribution of the $s \bar{s}$ component. When assuming this consideration to be valid up to energies of the $f_{0}(1710)$, one concludes that this state could be an unmixed glueball [51].

In the scalar-isodoublet sector (except for the well-established state $K_{0}^{*}(1430)$ ) the possible existence of a very broad meson in the 700-950 MeV region is discussed in recent years (see the review "Note on scalar mesons" in [1]). E.g., in some recent analyses the authors have found a pole which corresponds to this state $K_{0}^{*}(900)$ [52 58], while no such state was seen in the experiment performed by the BaBar Collaboration [59] and in the earlier analyses [60 62].

In view of all above circumstances, the problems connected with determining the nature of the observed mesonic states and their assignment to the quark-model configurations are still open in spite of a large amount of work devoted to these problems (see, e.g., Refs. 63 67]). It is clear that resonance parameters should be obtained, if possible, in a model-independent way. Here, we present results of the combined three-channel analysis of data on the processes $\pi \pi \rightarrow \pi \pi, K \bar{K}, \eta \eta, \eta \eta^{\prime}$ in the channel with the quantum numbers $I^{G} J^{P C}=0^{+} 0^{++}$. Study of the $K \pi$ scattering in the channel with $I\left(J^{P}\right)=\frac{1}{2}\left(0^{+}\right)$and the role of the strange scalar meson $K_{0}^{*}(900)(\kappa(800))$ goes beyond the scope of this paper and will be discussed in Ref. [68]. We have used a "model-independent" method [27, 30, 37, 39, 40] based on the first principles (analyticity and unitarity) directly applied to the analysis of experimental data. This approach permits us to omit a theoretical prejudice in extracting the resonance parameters. It is important that an uniformizingvariable method allows to avoid a model dependence when considering resonance contributions. This is possible since a main model-independent contribution of the resonance can be given by poles and corresponding zeros on a uniformization plane, whereas the possible remaining corrected and model-dependent contribution of the resonance is supposed to be taken into account in the background. This distinguishes substantially our model-independent method from the standard dispersion relation approach based also on analyticity and unitarity where, however, the model dependence arises inevitably when saturating dispersive integrals by the contributions of resonances. Then in our method, considering the obtained disposition of resonance poles on the Riemann surface, bearing witness to a relative strength of coupling with corresponding channels, and resonance masses, we draw conclusions about nature of the investigated states.

Unlike in the previous three-channel analysis of the above processes [25 27, 39], in this work we used a new uniformizing variable in which we took into account the left-hand branch-point at $s=0$ related to the thresholds of the $\pi \pi$ scattering in crossed channels, in addition to the right-hand branch-points related to the thresholds of the analyzed processes. This should diminish considerably dependence of the extracted parameters of resonances on the background because the elastic part of the $\pi \pi$ background is stipulated mainly by the contribution of the left-hand 
cuts.

The layout of the paper is as follows. In Sec. II we outline the two- and three-coupled channel formalism, where pole clusters on the Riemann surface are determined as characteristics of multichannel states and a classification of two- and three-channel resonances according to the types of the possible pole-clusters is given. We introduce also the new uniformizing variable for the three-channel case taking into account the left-hand branch-point at $s=0$, and show the disposition of the resonance poles and zeroes related to the various pole-clusters on the unifomization plane for the $\pi \pi$-scattering $S$-matrix element. In Sec. III we carry out the combined 3-channel analyses of data on the processes $\pi \pi \rightarrow \pi \pi, K \bar{K}, \eta \eta$ (variant I) and $\pi \pi \rightarrow \pi \pi, K \bar{K}, \eta \eta^{\prime}$ (variant II). In Sec. IV we summarize our conclusions, propose an assignment of the scalar mesons lying below $1.9 \mathrm{GeV}$ to lower nonets and discuss the obtained results.

\section{THE COUPLED-CHANNEL FORMALISM IN MODEL-INDEPENDENT APPROACH}

Our model-independent method which utilizes an uniformizing variable can be used only for the 2-channel case and under some conditions for the 3-channel one. Only in these cases we obtain a simple symmetric (easily to be interpreted) picture of the resonance poles and zeros of the $S$-matrix on the uniformization plane. The $S$-matrix is determined on the 4- and 8-sheeted Riemann surfaces for the 2- and 3-channel cases, respectively. The matrix elements $S_{\alpha \beta}$, where $\alpha, \beta=1,2,3$ denote the channels, have the right-hand cuts along the real axis of the complex $s$ plane ( $s$ is the invariant total energy squared), starting with the channel thresholds $s_{i}(i=1,2,3)$, and the left-hand cuts related to crossed channels. The Riemann-surface sheets are numbered according to the signs of the analytic continuations of the quantities $\sqrt{s-s_{\alpha}}$ as follows: in the 2-channel case

$$
\operatorname{signs}\left(\operatorname{Im} \sqrt{s-s_{1}}, \operatorname{Im} \sqrt{s-s_{2}}\right)=++,-+,--,+-
$$

correspond to sheets I, II, III, IV; in the 3-channel case

$$
\operatorname{signs}\left(\operatorname{Im} \sqrt{s-s_{1}}, \operatorname{Im} \sqrt{s-s_{2}}, \operatorname{Im} \sqrt{s-s_{3}}\right)=+++,-++,--+,+-+,+--,---,-+-,++-
$$

correspond to sheets I, II, - . VIII, respectively.

The resonance representations on the Riemann surfaces are obtained using formulas from Ref. [40] (see also Appendix), expressing analytic continuations of the $S$-matrix elements to unphysical sheets in terms of those on sheet I (physical) that have only the resonance zeros (beyond the real axis) around the physical region. Then, starting from the resonance zeros on sheet I one can obtain an arrangement of poles and zeros of resonances on the whole Riemann surface.

In the 2-channel case we obtain tree types of resonances described by a pair of conjugate zeros on sheet I only in $S_{11}$ - the type (a), only in $S_{22}-(\mathbf{b})$, and in each of $S_{11}$ and $S_{22}-(\mathbf{c})$. Then the formulas of the analytic continuations of the $S$-matrix elements to unphysical sheets [40] (see also Appendix) immediately give the resonance representation by poles and zeros on the 4-sheeted Riemann surface: to the resonances of types (a) and (b), there corresponds a pair of complex conjugate poles on sheet III shifted relative to a pair of poles on sheet II and IV, respectively. For the states of type (c) one must consider the corresponding two pairs of conjugate poles on sheet III.

In the 3-channel case we obtain seven types of resonances corresponding to seven possible situations when there are resonance zeros on sheet I only in $S_{11}-(\mathbf{a}) ; \quad S_{22}-(\mathbf{b}) ; \quad S_{33}-(\mathbf{c}) ; \quad S_{11}$ and $S_{22}-(\mathbf{d}) ; \quad S_{22}$ and $S_{33}-(\mathbf{e}) ; \quad S_{11}$ and $S_{33}-(\mathbf{f}) ; \quad S_{11}, S_{22}$, and $S_{33}-(\mathbf{g})$. Examples for the disposition of poles and zeros, corresponding to some of these types of the 3 -channel resonances, on the unifomization plane will be given in the next section.

The resonance of every type is represented by a pair of complex-conjugate clusters (of poles and zeros on the Riemann surface). The cluster type is related to the nature of the state. For example, if we consider the $\pi \pi, K \bar{K}$ and $\eta \eta$ channels, a resonance coupled relatively more strongly to the $\pi \pi$ channel than to the $K \bar{K}$ and $\eta \eta$ ones is described by the cluster of type (a). In the opposite case, it is represented by the cluster of type (e) (the state with a dominant $s \bar{s}$ component). The glueball must be represented by the cluster of type (g) (of type (c) in the 2-channel consideration) as a necessary condition for the ideal case, if this state lies above the thresholds of the considered channels.

A necessary and sufficient condition for existence of the multi-channel resonance is its representation by one of the types of pole clusters. Note that whereas cases (a), (b) and (c) can be simply related to the representation of resonances by multi-channel Breit-Wigner forms, cases (d), (e), (f) and (g) are practically lost in the Breit-Wigner description.

One can formulate a model-independent test as a necessary condition to distinguish a bound state of colorless particles (e.g., a $K \bar{K}$ molecule) and a $q \bar{q}$ bound state [37, 40, 69]. In the 1-channel case, the existence of the particle bound-state corresponds to the presence of a pole on the real axis under the threshold on the physical sheet. In the 
2-channel case the existence of the bound-state in channel 2 ( $K \bar{K}$ molecule) that, however, can decay into channel 1 ( $\pi \pi$ decay), would imply the presence of the pair of complex conjugate poles on sheet II under the second-channel threshold without the corresponding shifted pair of poles on sheet III.

In the 3 -channel case the bound state in channel $3(\eta \eta)$ that can decay into channels 1 ( $\pi \pi$ decay) and $2(K \bar{K}$ decay) is represented by the pair of complex conjugate poles on sheet II and by the pair of shifted poles on sheet III under the $\eta \eta$ threshold without the corresponding poles on sheets VI and VII. According to this test, an interpretation of the $f_{0}(980)$ as the $K \bar{K}$ molecule was rejected earlier [40]. The reason is that this state is represented by the cluster of type (a) in the 2-channel analysis of processes $\pi \pi \rightarrow \pi \pi, K \bar{K}$ and, therefore, does not satisfy the necessary condition to be the $K \bar{K}$ molecule. A further discussion of this topic will be given in the last section.

Unlike the standard dispersion relation approach in our model-independent method we use an advantage of the fact that the amplitude is a one-valued function on the Riemann surface. To this end a uniformizing variable is applied, which maps the Riemann surface onto a complex plane. This permit us to use the representation by the pole clusters very important for the broad multi-channel resonances. This is impossible in the standard dispersion relation and $K$-matrix approaches or in the Breit-Wigner one.

In the combined analysis of coupled processes it is convenient to use the Le Couteur-Newton relations 70 . They express the $S$-matrix elements of all coupled processes in terms of the Jost matrix determinant $d\left(\sqrt{s-s_{1}}, \cdots, \sqrt{s-s_{N}}\right)$ that is a real analytic function with the only square-root branch-points at $\sqrt{s-s_{\alpha}}=0$. The important branch points, corresponding to the thresholds of the coupled channels and to the crossing ones, are taken into account in the proper uniformizing variable. On the uniformization plane, the pole-cluster representation of the resonance is the good one.

It is obvious that the main model-independent contribution of resonances is factorized in the $S$-matrix elements from the background. The possible remaining corrected and model-dependent contributions of resonances are supposed to be included in the background. This is realized in a natural way: in the background, the corresponding elastic and inelastic phase shifts increase when some channel is opened. Therefore, we denote our approach as model independent.

In the 2-channel case, the $S$-matrix, determined on the 4-sheeted Riemann surface, can be uniformized using, e.g., the inverse Zhukovskij transformation [40] in which the thresholds of two channels are taken into account. Unfortunately, already in the 3-channel consideration a function determined on the 8-sheeted Riemann surface can be uniformized only on torus. This is unsatisfactory for our purposes. Therefore, we neglect the influence of the lowest $(\pi \pi)$ threshold branch-point at $s_{1}$ (however, unitarity on the $\pi \pi$-cut is taken into account). An approximation like this means the consideration of the nearest to the physical region semi-sheets of the Riemann surface of the $S$-matrix. In fact, we construct a 4-sheeted model of the initial 8-sheeted Riemann surface approximating it in accordance with our approach of a consistent account of the nearest singularities on all the relevant sheets. In practice, neglecting the influence of the $\pi \pi$-threshold branch-point means that we do not describe some small region near the threshold. Furthermore, we shall take into account in the uniformizing variable also the left-hand branch-point at $s=0$ related to the crossed $\pi \pi$ channels. The allowance for this branch-point should diminish the background dependence of the obtained results. As was indicated repeatedly (see, e.g., Ref. 11]) many analyses are subject to criticism (especially with the point of view of proof of the resonance existence) because the wide-resonance parameters are strongly controlled by the non-resonant background; this is particularly related to low-lying states. The allowance for the left-hand branch-point, related to the crossed channels, serves in part for a solution of this problem. For example in Ref. [30] a combined analysis of the processes $\pi \pi \rightarrow \pi \pi, K \bar{K}$ in the isoscalar-scalar sector was performed using the method of a uniformizing variable which includes two threshold branch-points and the left-hand one at $s=0$. In this respect a parameterless description of the $\pi \pi$ background was obtained due to the inclusion of the indicated left-hand branch-point. Moreover, it was shown that the large background, obtained in earlier analyses of the $S$-wave $\pi \pi$ scattering [40], hides in reality the $\sigma$-meson lying below $1 \mathrm{GeV}$.

In the 3-channel case the new uniformizing variable used below can have the form:

$$
w=\frac{\sqrt{\left(s-s_{2}\right) s_{3}}+\sqrt{\left(s-s_{3}\right) s_{2}}}{\sqrt{s\left(s_{3}-s_{2}\right)}}
$$

where we neglect the lowest $\pi \pi$-threshold branch-point and take into account the threshold branch-points related to two remaining channels and the left-hand branch-point at $s=0$. This variable maps our model of the 8-sheeted Riemann surface onto the $w$-plane divided into two parts by a unit circle centered at the origin. The semi-sheets I (III), II (IV), V (VII) and VI (VIII) are mapped onto the exterior (interior) of the unit disk in the 1st, 2nd, 3rd and 4th quadrants, respectively. The physical region extends from the point $\pi \pi$ on the imaginary axis (the first $\pi \pi$ threshold corresponding to $s_{1}$ ) along this axis down to the point $i$ on the unit circle (the second threshold corresponding to $s_{2}$ ). Then it extends further along the unit circle clockwise in the 1st quadrant to point 1 on the real axis (the third threshold corresponding to $\left.s_{3}\right)$ and then along the real axis to the point $b=\left(\sqrt{s_{2}}+\sqrt{s_{3}}\right) / \sqrt{s_{3}-s_{2}}$ into which $s=\infty$ is mapped on the $w$-plane. The intervals $(-\infty,-b],\left[-b^{-1}, b^{-1}\right],[b, \infty)$ on the real axis are the images of the corresponding edges of the left-hand cut of the $\pi \pi$-scattering amplitude. In Figs 1 and 2 , the 3-channel resonances of 
all the standard types in $S_{11}(w)$ are represented by the poles $(*)$ and zeroes $(\circ)$ symmetric to these poles with respect to the imaginary axis giving corresponding pole clusters. The "pole-zero" symmetry guarantees the elastic unitarity of $\pi \pi$ scattering in the $(\pi \pi, i)$ interval. For the data we use the results of phase analyses which are given for phase
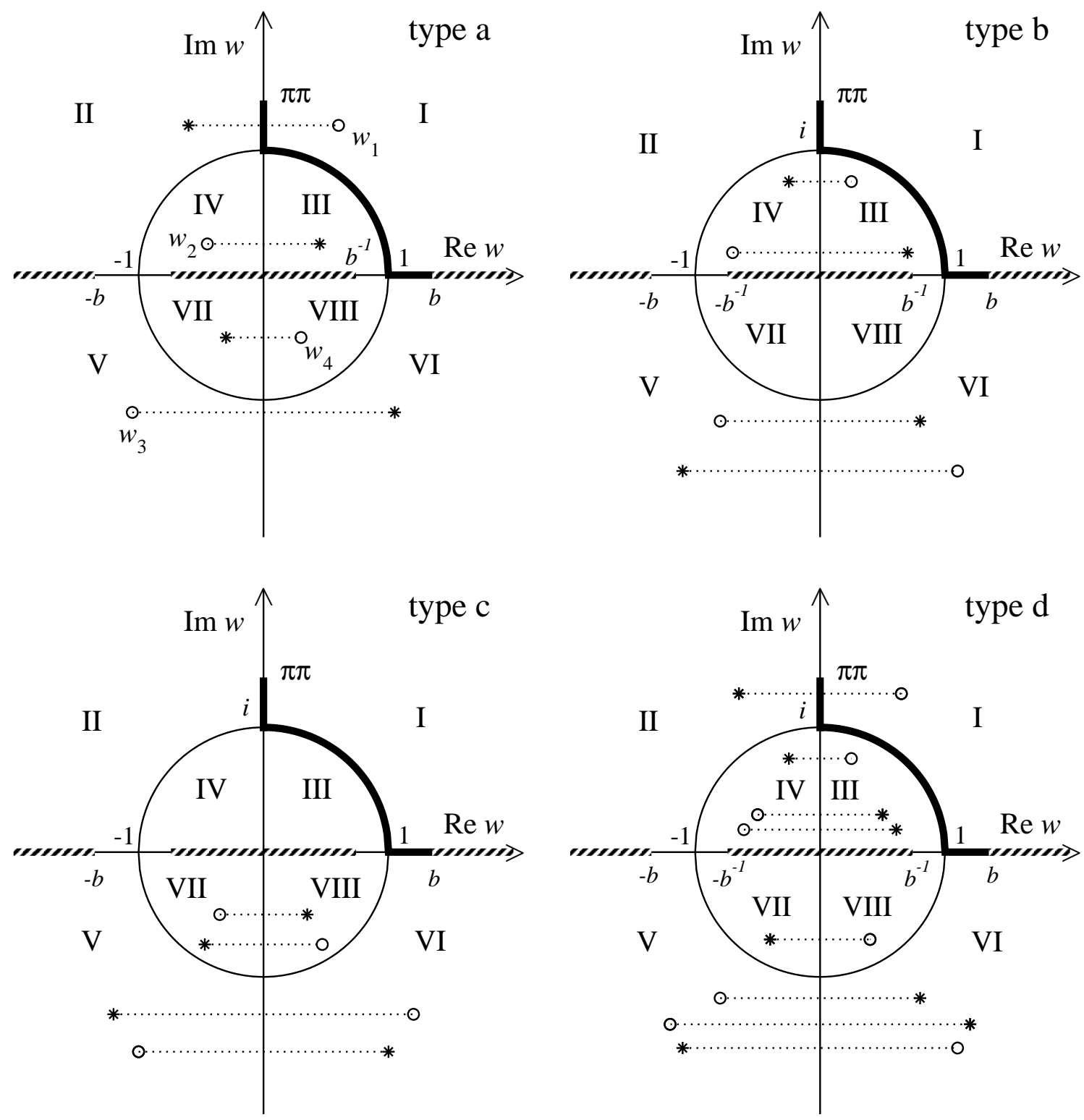

FIG. 1: Uniformization $w$-plane for the 3-channel- $\pi \pi$-scattering amplitude. Representation of resonances of types (a), (b), (c) and (d) is shown.

shifts of the amplitudes $\delta_{\alpha \beta}$ and for the modules of the $S$-matrix elements $\eta_{\alpha \beta}=\left|S_{\alpha \beta}\right|(\alpha, \beta=1,2,3)$ :

$$
S_{\alpha \alpha}=\eta_{\alpha \alpha} e^{2 i \delta_{\alpha \alpha}}, \quad S_{\alpha \beta}=\eta_{\alpha \beta} e^{i \phi_{\alpha \beta}}
$$

If below the third threshold there is the 2-channel unitarity then the relations

$$
\eta_{11}=\eta_{22}, \quad \eta_{12}=\left(1-\eta_{11}^{2}\right)^{1 / 2}, \quad \phi_{12}=\delta_{11}+\delta_{22}
$$

are fulfilled in this energy region. 

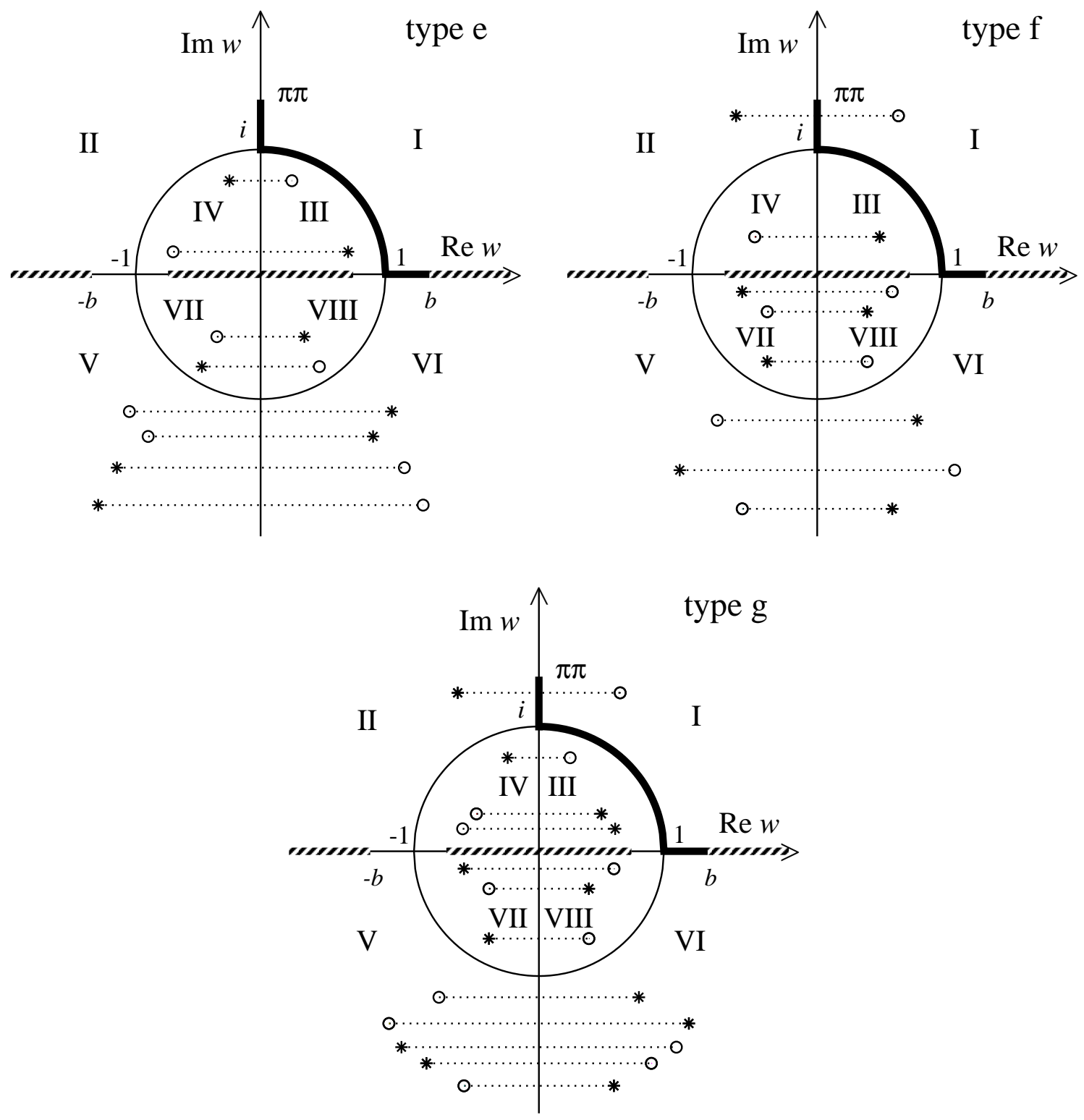

FIG. 2: Uniformization $w$-plane for the 3-channel scattering amplitude. Representation of resonances of types (e), (f), and (g) is shown.

Masses and total widths can be calculated using the denominator of formula for the resonance part of the amplitude of the form

$$
T^{r e s}=\frac{\sqrt{s} \Gamma_{e l}}{m_{r e s}^{2}-s-i \sqrt{s} \Gamma_{t o t}}
$$

taking the pole positions on sheets II, IV and VIII because, as one can see in Ref. [40], the analytic continuations of the corresponding $S$-matrix elements on these sheets only have the forms

$$
\propto 1 / S_{11}^{\mathrm{I}}, \quad \propto 1 / S_{22}^{\mathrm{I}} \text { and } \propto 1 / S_{33}^{\mathrm{I}},
$$

respectively. This means that the pole positions of resonances only on these sheets are at the same points in the complex-energy plane as the resonance zeros on the physical sheet and are not shifted due to the coupling of channels. Note that the poles on indicated sheets are not always nearest to the physical region. 


\section{ANALYSIS OF THE ISOSCALAR-SCALAR SECTOR}

We analyzed the isoscalar $S$-waves of the processes

$$
\pi \pi \rightarrow \pi \pi, K \bar{K}, \eta \eta, \eta \eta^{\prime} .
$$

The experimental data on the $\pi \pi$ scattering from $0.575 \mathrm{GeV}$ to $1.89 \mathrm{GeV}$ are taken from Ref. 71] and below $1 \mathrm{GeV}$ from the works 72 74]. For $\pi \pi \rightarrow K \bar{K}$ the data 75$]$ from threshold to about $1.6 \mathrm{GeV}$ are used. The data for $\pi \pi \rightarrow \eta \eta$ up to $1.72 \mathrm{GeV}$ are taken from Ref. [76] and for $\pi \pi \rightarrow \eta \eta^{\prime}$ from threshold to $1.81 \mathrm{GeV}$ from Ref. [77].

In the model-independent approach we have performed two variants of the 3-channel analysis: variant I - the combined analysis of processes $\pi \pi \rightarrow \pi \pi, K \bar{K}, \eta \eta$; variant II - analysis of $\pi \pi \rightarrow \pi \pi, K \bar{K}, \eta \eta^{\prime}$. The influence of the $\eta \eta^{\prime}$-channel in the case I and of $\eta \eta$ in the case II are taken into account via the background. The analysis has been carried out with the new uniformizing variable (3) $\left(s_{3}\right.$ is $4 m_{\eta}^{2}$ in variant I and $\left(m_{\eta}+m_{\eta^{\prime}}\right)^{2}$ in variant II; in the following the quantities related to variant II are primed).

On the $w$-plane the Le Couteur-Newton relations have the form [40]:

$$
\begin{aligned}
& S_{11}=\frac{d^{*}\left(-w^{*}\right)}{d(w)}, \quad S_{22}=\frac{d\left(-w^{-1}\right)}{d(w)}, \quad S_{33}=\frac{d\left(w^{-1}\right)}{d(w)} \\
& S_{11} S_{22}-S_{12}^{2}=\frac{d^{*}\left(w^{*-1}\right)}{d(w)}, \quad S_{11} S_{33}-S_{13}^{2}=\frac{d^{*}\left(-w^{*-1}\right)}{d(w)}, \quad S_{22} S_{33}-S_{23}^{2}=\frac{d(-w)}{d(w)} .
\end{aligned}
$$

In this case the subscripts in the matrix elements $S_{\alpha \beta}$ denote $\alpha, \beta=1-\pi \pi, 2-K \bar{K}, 3-\eta \eta$ or $\eta \eta^{\prime}$.

The $S$-matrix elements in relations (7) are taken as the products $S=S_{B} S_{\text {res }}$ where $S_{B}$ describes the background and $S_{r e s}$ the resonance contributions. The d-function for the resonance part is

$$
d_{r e s}(w)=w^{-\frac{M}{2}} \prod_{r=1}^{M}\left(w+w_{r}^{*}\right)
$$

where $M$ is the number of resonance zeros. For the background part $S_{B}$ the $d$-function has the following form:

$$
\begin{gathered}
d_{B}=\exp \left[-i\left(a+\sum_{n=1}^{3} \frac{\sqrt{s-s_{n}}}{2 m_{n}}\left(\alpha_{n}+i \beta_{n}\right)\right)\right] \\
\alpha_{n}=a_{n 1}+a_{n \sigma} \frac{s-s_{\sigma}}{s_{\sigma}} \theta\left(s-s_{\sigma}\right)+a_{n v} \frac{s-s_{v}}{s_{v}} \theta\left(s-s_{v}\right), \\
\beta_{n}=b_{n 1}+b_{n \sigma} \frac{s-s_{\sigma}}{s_{\sigma}} \theta\left(s-s_{\sigma}\right)+b_{n v} \frac{s-s_{v}}{s_{v}} \theta\left(s-s_{v}\right)
\end{gathered}
$$

where $s_{\sigma}$ is the $\sigma \sigma$ threshold, $s_{v}$ the combined threshold of many opened channels in the range of $\sim 1.5 \mathrm{GeV}$ $\left(\eta \eta^{\prime}, \rho \rho, \omega \omega\right)$. These threshold are determined in the analysis.

In variant II, the terms

$$
a_{n \eta}^{\prime} \frac{s-4 m_{\eta}^{2}}{4 m_{\eta}^{2}} \theta\left(s-4 m_{\eta}^{2}\right) \quad \text { and } \quad b_{n \eta}^{\prime} \frac{s-4 m_{\eta}^{2}}{4 m_{\eta}^{2}} \theta\left(s-4 m_{\eta}^{2}\right)
$$

were added to $\alpha_{n}^{\prime}$ and $\beta_{n}^{\prime}$, respectively, to account for an influence of the $\eta \eta$-channel.

In the analysis, we included all the five resonances discussed below $1.9 \mathrm{GeV}$ in the PDG issue [1]. In variant I, for the $\pi \pi$-scattering and $\pi \pi \rightarrow K \bar{K}$, we considered the data for phase shifts and modules of the $S$-matrix elements in the energy regions from about 0.4 to $1.89 \mathrm{GeV}$ and from the threshold to about $1.6 \mathrm{GeV}$, respectively; for $\pi \pi \rightarrow \eta \eta$, the data for the squared module of the $S$-matrix element from the threshold to $1.72 \mathrm{GeV}$. A satisfactory description has been obtained. Furthermore it was found [78 that the data on the $\pi \pi$ scattering below $1 \mathrm{GeV}$ admit two solutions. Let us call them, as in the indicated work [78], "up" and "down" because the $\pi \pi$-scattering phase shift goes a bit higher in the first case than in the second case. In variant I, for the "up" solution, we considered the representation of resonances by different pole-clusters that are admitted by the data. For the "down" solution, we show the formally best case. In all cases, the $f_{0}(600)$ is represented by the pole cluster of type $(\mathbf{a})$, the $f_{0}(980)$ is represented only by 
TABLE I: Variant I: the quality of description of the data for the best variants of representation of considered states obtained in the analysis. The letters in the second column denote the pole clusters describing respectively resonances $f_{0}(1370), f_{0}(1500)$ and $f_{0}(1710)$.

\begin{tabular}{|c|c|c|c|c|c|}
\hline Solution & & $\begin{array}{c}\pi \pi \text { scattering } \\
\chi^{2} / \text { dof } \\
\end{array}$ & $\begin{array}{c}\pi \pi \rightarrow K \bar{K} \\
\chi^{2} / \text { dof } \\
\end{array}$ & $\begin{array}{c}\pi \pi \rightarrow \eta \eta \\
\chi^{2} / \mathrm{ndp}\end{array}$ & $\begin{array}{c}\text { The total } \\
\chi^{2} / \text { dof }\end{array}$ \\
\hline \multirow{3}{*}{ "up" } & bgb & $155.784 /(169-35) \approx 1.16$ & $148.702 /(120-33) \approx 1.71$ & 1.02 & $320.770 /(305-42) \approx 1.22$ \\
\hline & bgc & $146.684 /(169-35) \approx 1.09$ & $152.274 /(120-33) \approx 1.75$ & 0.99 & $314.754 /(305-42) \approx 1.20$ \\
\hline & $\operatorname{cgc}$ & $143.269 /(169-35) \approx 1.07$ & $154.469 /(120-33) \approx 1.78$ & 0.91 & $312.365 /(305-42) \approx 1.19$ \\
\hline
\end{tabular}

the pole on sheet II and shifted pole on sheet III; the resonances $f_{0}(1370)$ and $f_{0}(1710)$ can be described by the pole clusters of type $(\mathbf{b})$ or $(\mathbf{c}) ; f_{0}(1500)$, of type $(\mathbf{g})$.

In Table I, we demonstrate quality of description for each separate process in the frame of the best combined description of all three processes for various acceptable variants of representation of considered states. We use abbreviations "dof" - number of degrees of freedom and "ndp" - number of data points.

It is clear that one should achieve the best description of the separate process; however, then the combined description of all three processes would be worse.

In Tables II and III there are given the masses and total widths of states for the indicated cases, calculated from the pole positions on sheets II, IV and VIII for resonances of types (a),(b)and (c), respectively, using denominator of the resonance part of amplitude in the form (6). For the resonance $f_{0}(1500)$ of type (g), the poles can be used on all indicated sheets.

TABLE II: The "up" solution: the masses and total widths (in MeV) of the $f_{0}$ resonances, obtained at analyzing for acceptable variants of representation of considered states. The letters in the upper row denote the pole clusters describing respectively resonances $f_{0}(1370), f_{0}(1500)$ and $f_{0}(1710)$.

\begin{tabular}{|c|c|c|c|c|c|c|c|c|}
\hline \multirow[b]{2}{*}{ State } & \multicolumn{2}{|c|}{$\mathrm{bgb}$} & \multicolumn{2}{|c|}{$\mathrm{cgb}$} & \multicolumn{2}{|c|}{$\mathrm{bgc}$} & \multicolumn{2}{|c|}{$\mathrm{cgc}$} \\
\hline & $m_{r e s}$ & $\Gamma_{t o t}$ & $m_{\text {res }}$ & $\Gamma_{t o t}$ & $m_{r e s}$ & $\Gamma_{t o t}$ & $m_{r e s}$ & $\Gamma_{t o t}$ \\
\hline$f_{0}(600)$ & $713.7 \pm 5.4$ & $627.0 \pm 7.2$ & $735.0 \pm 6.1$ & $686.0 \pm 7.0$ & $627.0 \pm 7.3$ & $665.8 \pm 11.0$ & $604.5 \pm 5.7$ & $567.0 \pm 5.4$ \\
\hline$f_{0}(980)$ & $1007.6 \pm 2.2$ & $45.2 \pm 2.8$ & $1007.1 \pm 2.6$ & $50.6 \pm 2.8$ & $1007.3 \pm 1.9$ & $50.8 \pm 2.8$ & $1004.7 \pm$ & $54.2 \pm 2.8$ \\
\hline$f_{0}(1370)$ & $1404.0 \pm 7.0$ & $279.1 \pm 22.0$ & $1390.5 \pm 14.3$ & $223.4 \pm 42.8$ & $1325.6 \pm 11.1$ & $344.6 \pm 24.4$ & $1374.5 \pm 16.7$ & $322.0 \pm 60.8$ \\
\hline$f_{0}(1500)$ & $1532.6 \pm 15.9$ & $648.2 \pm 26.6$ & $1544.9 \pm 12.2$ & $646.2 \pm 26.0$ & $1556.6 \pm 13.5$ & $690.4 \pm 28.6$ & $1535.4 \pm 12.3$ & $671.4 \pm 26.8$ \\
\hline$f_{0}(1710)$ & $1750.9 \pm 35.6$ & $118.2 \pm 30.2$ & $1751.0 \pm 23.8$ & $118.0 \pm 50.8$ & $1759.2 \pm 755.7$ & $207.0 \pm 420.3$ & $1759.2 \pm 716.4$ & $201.8 \pm 385.8$ \\
\hline
\end{tabular}

TABLE III: The "down" solution: the masses and total widths (in $\mathrm{MeV}$ ) of the $f_{0}$ resonances, obtained at analyzing for the case when the resonances $f_{0}(1370), f_{0}(1500)$ and $f_{0}(1710)$ are described by the pole clusters of type (c), (g) and (c), respectively.

\begin{tabular}{ccc}
\hline \hline State & $m_{\text {res }}$ & $\Gamma_{\text {tot }}$ \\
\hline$f_{0}(600)$ & $769.0 \pm 10.0$ & $1036.9 \pm 11.8$ \\
$f_{0}(980)$ & $1007.2 \pm 3.1$ & $64.6 \pm 3.0$ \\
$f_{0}(1370)$ & $1396.4 \pm 24.7$ & $355.2 \pm 79.6$ \\
$f_{0}(1500)$ & $1534.1 \pm 9.2$ & $636.6 \pm 25.8$ \\
$f_{0}(1710)$ & $1731.0 \pm 43.6$ & $203.4 \pm 34.8$ \\
\hline \hline
\end{tabular}

As to a quality of description, it is impossible to select any of the above-indicated solutions on the basis of analyzing jointly only three considered processes. It is required to add in the combined analysis also relevant processes of 
decay. We selected the "up" solution mainly because its parameters of the $f_{0}(600)$ remarkably accord with prediction $\left(m_{\sigma} \approx m_{\rho}\right.$ and $\left.\Gamma_{t o t} \approx 600 \mathrm{MeV}\right)$ by Weinberg [6], however, for now we should consider both solutions. Furthermore, we take a scenario in which the $f_{0}(1370), f_{0}(1500)$ and $f_{0}(1710)$ are described by the pole clusters of type (c), (g) and (b), respectively. The point is that the parameters of the $f_{0}(1500)$ can be calculated from the pole positions on sheets II, IV and VIII. Therefore, an additional criterion for self-consistency of results is a mutual closeness of values of the obtained parameters of this important state on the indicated sheets. According to this criterion the selected scenario is the most relevant.

In Tables $\mathbb{\square}$ and $\nabla$ we show the obtained pole clusters for resonances in the complex energy plane $\sqrt{s}$, corresponding to the cases when the $f_{0}(1370), f_{0}(1500)$ and $f_{0}(1710)$ are described by the pole clusters of the type (c), (g) and (b) for the "up" and of type (c), (g) and (c) for "down" solutions, respectively. The poles corresponding to the $f_{0}(1500)$ on sheets IV, VI, and VIII are of the 2nd order and those on the sheet V of the 3rd order in our approximation.

TABLE IV: The pole clusters for the $f_{0}$-resonances for the "up" solution in variant I. $\sqrt{s_{r}}=\mathrm{E}_{r}+i \Gamma_{r} / 2$ in $\mathrm{MeV}$ is given.

\begin{tabular}{|c|c|c|c|c|c|c|c|c|}
\hline Sheet & & II & III & IV & $\mathrm{V}$ & VI & VII & VIII \\
\hline \multirow[t]{2}{*}{$f_{0}(600)$} & $\mathrm{E}_{r}$ & $650.1 \pm 6.6$ & $703.8 \pm 10.9$ & & & $655.9 \pm 27.6$ & $602.2 \pm 22.0$ & \\
\hline & $\Gamma_{r} / 2$ & $343.0 \pm 3.5$ & $343.0 \pm 3.5$ & & & $343.0 \pm 3.5$ & $343.0 \pm 3.5$ & \\
\hline \multirow[t]{2}{*}{$f_{0}(980)$} & $\mathrm{E}_{r}$ & $1006.8 \pm 2.6$ & $980.8 \pm 3.8$ & & & & & \\
\hline & $\Gamma_{r} / 2$ & $25.3 \pm 1.4$ & $37.2 \pm 2.2$ & & & & & \\
\hline \multirow[t]{2}{*}{$f_{0}(1370)$} & $\mathrm{E}_{r}$ & & & & $1386.0 \pm 14.2$ & $1386.0 \pm 14.2$ & $1386.0 \pm 14.2$ & $1386.0 \pm 14.2$ \\
\hline & $\Gamma_{r} / 2$ & & & & $182.9 \pm 34.2$ & $179.5 \pm 33.6$ & $155.7 \pm 18$ & $111.7 \pm 21.4$ \\
\hline \multirow[t]{2}{*}{$f_{0}(1500)$} & $\mathrm{E}_{r}$ & $1510.7 \pm 12.2$ & $1530.0 \pm 12.7$ & $1510.7 \pm 12.2$ & $1510.6 \pm 8.5$ & $1513 . \pm 5.8$ & $1486.4 \pm 14.6$ & $1510.7 \pm 12.2$ \\
\hline & $\Gamma_{r} / 2$ & $323.1 \pm 13.0$ & $164.7 \pm 11.0$ & $290.5 \pm 46.2$ & $156.9 \pm 9.0$ & $193.2 \pm 6.4$ & $134.4 \pm 21.6$ & $332.9 \pm 73.7$ \\
\hline \multirow[t]{2}{*}{$\overline{f_{0}(1710)}$} & $\mathrm{E}_{r}$ & & $1750.0 \pm 23.8$ & $1750.0 \pm 23.8$ & $1750.0 \pm 23.8$ & $1750.0 \pm 23.8$ & & \\
\hline & $\Gamma_{r} / 2$ & & $65.2 \pm 26.6$ & $59.0 \pm 25.4$ & $63.0 \pm 23.6$ & $69.2 \pm 24.0$ & & \\
\hline
\end{tabular}

TABLE V: The pole clusters for the $f_{0}$-resonances for the "down" solution in variant I. $\sqrt{s_{r}}=\mathrm{E}_{r}+i \Gamma_{r} / 2$ in $\mathrm{MeV}$ is given.

\begin{tabular}{|c|c|c|c|c|c|c|c|c|}
\hline Sheet & & II & III & IV & $\mathrm{V}$ & VI & VII & VIII \\
\hline \multirow[t]{2}{*}{$f_{0}(600)$} & $\mathrm{E}_{r}$ & $567.9 \pm 12.4$ & $642.0 \pm 17.7$ & & & $647.7 \pm 29.1$ & $573.6 \pm 25.5$ & \\
\hline & $\Gamma_{r} / 2$ & $518.5 \pm 5.9$ & $518.5 \pm 5.9$ & & & $518.5 \pm 5.9$ & $518.5 \pm 5.9$ & \\
\hline \multirow[t]{2}{*}{$f_{0}(980)$} & $\mathrm{E}_{r}$ & $1006.7 \pm 3.1$ & $970.1 \pm 5.8$ & & & & & \\
\hline & $\Gamma_{r} / 2$ & $32.3 \pm 1.5$ & $55.4 \pm 2.6$ & & & & & \\
\hline \multirow[t]{2}{*}{$f_{0}(1370)$} & $\mathrm{E}_{r}$ & & & & $1385.1 \pm 24.4$ & $1385.1 \pm 24.4$ & $1385.1 \pm 24.4$ & $1385.1 \pm 24.4$ \\
\hline & $\Gamma_{r} / 2$ & & & & $287.0 \pm 73.7$ & $267.4 \pm 83.1$ & $158.0 \pm 41.8$ & $177.6 \pm 39.8$ \\
\hline \multirow[t]{2}{*}{$f_{0}(1500)$} & $\mathrm{E}_{r}$ & $1500.7 \pm 4.9$ & $1495.0 \pm 9.0$ & $1500.7 \pm 4.9$ & $1496.7 \pm 7.2$ & $1510.2 \pm 4.6$ & $1501.2 \pm 9.8$ & $1500.7 \pm 4.9$ \\
\hline & $\Gamma_{r} / 2$ & $318.3 \pm 12.9$ & $133.6 \pm 10.6$ & $231.9 \pm 17.6$ & $141 . \pm 6.3$ & $185.2 \pm 4.0$ & $99 . \pm 18.0$ & $345.9 \pm 14.5$ \\
\hline \multirow[t]{2}{*}{$f_{0}(1710)$} & $\mathrm{E}_{r}$ & & & & $1728.0 \pm 43.7$ & $1728.0 \pm 43.7$ & $1728.0 \pm 43.7$ & $1728.0 \pm 43.7$ \\
\hline & $\Gamma_{r} / 2$ & & & & $139.9 \pm 69.0$ & $138.7 \pm 8.9$ & $100.5 \pm 48.4$ & $101.7 \pm 17.4$ \\
\hline
\end{tabular}

The background parameters are: "up" solution $-a=0.4704 \pm 0.0364, a_{11}=-0.2376 \pm 0.0132, a_{1 \sigma}=0.186 \pm 0.0335$, $a_{1 v}=-0.0788 \pm 0.0535, b_{11}=b_{1 \sigma}=0, b_{1 v}=0.0305 \pm 0.0112, a_{21}=-1.7768 \pm 0.0461, a_{2 \sigma}=0.5204 \pm 0.0254$, $a_{2 v}=-9.22 \pm 0.649, b_{21}=0.0132 \pm 0.0131, b_{2 \sigma}=0, b_{2 v}=7.385 \pm 1.354, b_{31}=0.5494 \pm 0.0458, b_{3 \sigma}=0.8995 \pm 0.0997$, $b_{3 v}=0 ; s_{\sigma}=1.638 \mathrm{GeV}^{2}, s_{v}=2.085 \mathrm{GeV}^{2}$; "down" solution $-a=0.2431 \pm 0.0322, a_{11}=-0.0553 \pm 0.0113$, $a_{1 \sigma}=0.0914 \pm 0.0103, a_{1 v}=-0.0478 \pm 0.0098, b_{11}=b_{1 \sigma}=0, b_{1 v}=0.0469 \pm 0.0104, a_{21}=-1.6811 \pm 0.0426$, $a_{2 \sigma}=-0.247 \pm 0.1987, a_{2 v}=-7.2 \pm 0.5858, b_{21}=0.0329 \pm 0.0131, b_{2 \sigma}=0, b_{2 v}=7.765 \pm 1.4301, b_{31}=0.6135 \pm 0.0495$, $b_{3 \sigma}=0.6617 \pm 0.1099, b_{2 v}=0$.

The obtained zero positions of the resonances on the $w$-plane are: 
"up" solution -

$$
\begin{array}{rll}
\text { for } f_{0}(600): & w_{1}=3.8538993+4.1546473 i, & w_{2}=-0.1320698+0.1400331 i, \\
\text { for } f_{0}(980): & w_{3}=-3.8192504-4.1137414 i, & w_{4}=0.1109493-0.1194408 i, \\
\text { for } f_{0}(1370): & w_{5}=0.6671266+1.1471002 i, & w_{6}=-0.2290429+0.5613030 i, \\
& w_{7}=-3.0340738-0.4551088 i, & w_{8}=3.0385254-0.4624306 i, \\
\text { for } f_{0}(1500): & w_{9}=-0.3346148-0.0335710 i, & w_{10}=0.3351386-0.0326940 i, \\
w_{11}=3.4148479+0.5291918 i, & w_{12}=0.2899891+0.0420199 i, \\
w_{13}=-0.2847651-0.0449140 i, & w_{14}=w_{15}=-0.3016876+0.0264597 i \\
w_{16}=w_{17}=w_{18}=-3.2842692-0.3472515 i, & \\
w_{19}=w_{20}=3.2517666-0.2894345 i, & w_{21}=w_{22}=0.3110365-0.0259720 i, \\
\text { for } f_{0}(1710): & w_{23}=0.2818700+0.0051870 i, & w_{24}=-0.2817805+0.0057246 i . \\
w_{25}=-3.5479990-0.0764492 i, & w_{26}=3.5470806-0.0696555 i ;
\end{array}
$$

\begin{tabular}{|c|c|c|c|c|c|c|c|c|}
\hline Sheet & & II & III & IV & $\mathrm{V}$ & VI & VII & VIII \\
\hline \multirow{2}{*}{$f_{0}(600)$} & $\mathrm{E}_{r}^{\prime}$ & $558.7 \pm 13.3$ & $564.3 \pm 13.7$ & & & $541.3 \pm 55.0$ & $535.7 \pm 26.0$ & \\
\hline & $\Gamma_{r}^{\prime} / 2$ & $529 \pm 17.4$ & $529 \pm 17.4$ & & & $529 \pm 17.4$ & $529 \pm 17.4$ & \\
\hline \multirow[t]{2}{*}{$f_{0}(980)$} & $\mathrm{E}_{r}^{\prime}$ & $1009.0 \pm 3.1$ & $986.1 \pm 5.5$ & & & & & \\
\hline & $\Gamma_{r}^{\prime} / 2$ & $31.8 \pm 1.8$ & $57.4 \pm 2.9$ & & & & & \\
\hline \multirow[t]{2}{*}{$f_{0}(1370)$} & $\mathrm{E}_{r}^{\prime}$ & & $1411.6 \pm 8.1$ & $1411.6 \pm 8.1$ & $1428.4 \pm 11.0$ & $1428.4 \pm 11.0$ & & \\
\hline & $\Gamma_{r}^{\prime} / 2$ & & $215.6 \pm 21.2$ & $235 \pm 22.6$ & $235 \pm 22.6$ & $215.6 \pm 21.2$ & & \\
\hline \multirow[t]{2}{*}{$f_{0}(1500)$} & $\mathrm{E}_{r}^{\prime}$ & $1496.9 \pm 4.7$ & $1503.0 \pm 3.9$ & $1496.9 \pm 4.7$ & $1496.9 \pm 4.7$ & $1494.6 \pm 2.9$ & $1496.9 \pm 4.7$ & \\
\hline & $\Gamma_{r}^{\prime} / 2$ & $198.5 \pm 7.8$ & $236.0 \pm 5.7$ & $193.1 \pm 8.6$ & $198.5 \pm 7.8$ & $193.7 \pm 4.7$ & $193.1 \pm 8.6$ & \\
\hline \multirow[t]{2}{*}{$f_{0}(1710)$} & $\mathrm{E}_{r}^{\prime}$ & & & & $1743.0 \pm 17.8$ & $1743.0 \pm 17.8$ & $1743.0 \pm 17.8$ & $1743.0 \pm 17.8$ \\
\hline & $\Gamma_{r}^{\prime} / 2$ & & & & $144.1 \pm 40.3$ & $111.5 \pm 31.9$ & $82.1 \pm 36.6$ & $114.7 \pm 38.6$ \\
\hline
\end{tabular}

"down" solution -

$$
\begin{array}{rcl}
\text { for } f_{0}(600): & w_{1}=5.2482964+3.3618022 i, & w_{2}=-0.1459568+0.0959815 i, \\
\text { for } f_{0}(980): & w_{3}=-4.7510997-3.1267321 i, & w_{4}=0.1358737-0.0873064 i, \\
\text { for } f_{0}(1370): & w_{5}=0.7366709+1.2022465 i, & w_{6}=-0.2172355+0.4780125 i, \\
& w_{7}=-3.1626888-0.6250175 i, & w_{8}=3.1950376-0.6565649 i, \\
\text { for } f_{0}(1500): & w_{9}=-0.3228711-0.0481947 i, & w_{10}=0.3267097-0.0444134 i, \\
& w_{11}=3.3973448+0.5354069 i, & w_{12}=0.2981317+0.0378036 i, \\
w_{16}=-0.2837024-0.0468876 i, & w_{14}=w_{15}=-0.3096900+0.0250295 i, \\
& w_{17}=w_{18}=-3.2730730-0.3367541 i, & \\
w_{19}=w_{20}=3.2161986-0.2715142 i, & w_{21}=w_{22}=0.3114429-0.0186598 i, \\
\text { for } f_{0}(1710): & w_{23}=-3.5403847-0.1579625 i, & w_{24}=3.5407727-0.1592757 i . \\
w_{25}=-0.2829782-0.0093685 i, & w_{26}=0.2830083-0.0092620 i .
\end{array}
$$

For variant II we got the following description: for $\pi \pi$ scattering $\chi^{2} /$ dof $=148.786 /(169-30) \approx 1.07$; for $\pi \pi \rightarrow K \bar{K}$ $\chi^{2} /$ dof $=155.006 /(120-29) \approx 1.70$; for $\pi \pi \rightarrow \eta \eta^{\prime} \chi^{2} / \mathrm{ndp} \approx 0.3$. The total $\chi^{2} /$ dof is $306.187 /(297-37) \approx 1.18$. In this case the $f_{0}(600)$ is described by the cluster of type $\left(\mathbf{a}^{\prime}\right) ; f_{0}(1370)$, type $\left(\mathbf{b}^{\prime}\right) ; f_{0}(1500)$, type $\left(\mathbf{d}^{\prime}\right) ; f_{0}(1710)$, type $\left(\mathbf{c}^{\prime}\right)$. In Table V1] we indicate the obtained pole clusters for resonances on the eight sheets in the complex energy plane $\sqrt{s}$. The poles on sheets IV and V, corresponding to the $f_{0}(1500)$, are of the 2 nd order (this is an approximation).

TABLE VI: The pole clusters for the $f_{0}$-resonances in variant II. $\sqrt{s_{r}^{\prime}}=\mathrm{E}_{r}^{\prime}+i \Gamma_{r}^{\prime} / 2$ in MeV is given.

The background parameters are: $a^{\prime}=0.2315 \pm 0.0085, a_{11}^{\prime}=0, a_{1 \eta}^{\prime}=-0.0616 \pm 0.0321, a_{1 \sigma}^{\prime}=0.0298 \pm 0.0876$, $a_{1 v}^{\prime}=0.0622 \pm 0.0703, b_{11}^{\prime}=b_{1 \eta}^{\prime}=b_{1 \sigma}^{\prime}=0, b_{1 v}^{\prime}=0.0449 \pm 0.0105, a_{21}^{\prime}=-3.1359 \pm 0.0628, a_{2 \eta}^{\prime}=0, a_{2 \sigma}^{\prime}=$ 
$0.4866 \pm 0.2778, a_{2 v}^{\prime}=-4.532 \pm 0.7199, b_{21}^{\prime}=0, b_{2 \eta}^{\prime}=-0.7478 \pm 0.0607, b_{2 \sigma}^{\prime}=2.5545 \pm 0.2067, b_{2 v}^{\prime}=1.948 \pm 1.785$, $b_{31}^{\prime}=0.4489 \pm 0.0606, s_{\sigma}=1.638 \mathrm{GeV}^{2}, s_{v}=2.126 \mathrm{GeV}^{2}$.

The obtained zero positions of the resonances on the $w^{\prime}$-plane are:

$$
\begin{array}{rcl}
\text { for } f_{0}(600): & w_{1}^{\prime}=2.8974465+2.0075214 i, & w_{2}^{\prime}=-0.2343026+0.1631268 i, \\
& w_{3}^{\prime}=-2.9703646-2.0251533 i, & w_{4}^{\prime}=0.2287780-0.1551171 i, \\
\text { for } f_{0}(980): & w_{5}^{\prime}=0.3793973+1.1246624 i, & w_{6}^{\prime}=-0.2267376+0.6892463 i, \\
\text { for } f_{0}(1370): & w_{7}^{\prime}=0.6466646+0.2560839 i, & w_{8}^{\prime}=-0.6616945+0.2603249 i, \\
& w_{9}^{\prime}=-1.3218615-0.4907520 i, & w_{10}^{\prime}=1.3490514-0.5056393 i, \\
\text { for } f_{0}(1500): & w_{11}^{\prime}=1.3583321+0.3852687 i, & w_{12}^{\prime}=0.6855763+0.1928257 i, \\
& w_{13}^{\prime}=1.3583321-0.3852687 i, & w_{14}^{\prime}=0.6855763-0.1928257 i, \\
w_{15}^{\prime}= & w_{16}^{\prime}=-0.6543233+0.1907843 i, w_{17}^{\prime}= & w_{18}^{\prime}=-1.3502545-0.3835908 i, \\
\text { for } f_{0}(1710): & w_{19}^{\prime}=-1.5488743-0.1115869 i, & w_{20}^{\prime}=1.5602461-0.1413653 i, \\
& w_{21}^{\prime}=-0.6417003-0.0474415 i, & w_{22}^{\prime}=0.6471581-0.0350139 i .
\end{array}
$$

Masses and total widths of the states, calculated from the pole positions on sheets II , IV and VIII for resonances of types (a),(b)and (c), respectively, and on sheets II or IV for resonance of type (d), are presented in Table VII.

TABLE VII: Variant II: the masses and total widths of the $f_{0}$ resonances.

\begin{tabular}{ccc}
\hline \hline State & $m_{r e s}^{\prime}[\mathrm{MeV}]$ & $\Gamma_{\text {tot }}^{\prime}[\mathrm{MeV}]$ \\
\hline$f_{0}(600)$ & $769.4 \pm 15.4$ & $1058.0 \pm 34.8$ \\
$f_{0}(980)$ & $1009.5 \pm 3.1$ & $63.6 \pm 3.6$ \\
$f_{0}(1370)$ & $1431.0 \pm 8.8$ & $469.9 \pm 45.2$ \\
$f_{0}(1500)$ & $1510.1 \pm 4.8$ & $397.1 \pm 15.6$ \\
$f_{0}(1710)$ & $1746.8 \pm 17.9$ & $229.4 \pm 77.2$ \\
\hline \hline
\end{tabular}

In Figures 35, we show results of fitting to the experimental data in both variants.

Note, that the "up" solution is not revealed in variant II when in the analysis the data both below and above the $K \bar{K}$-threshold are used. This suggests that, the $\eta \eta$-threshold branch-point must be taken into account explicitly. It is not sufficient to consider influence of the $\eta \eta$ channel only via the background.

To learn more on existence of the $f_{0}(1370)$ (see the discussion in Introduction), we considered a possibility of description in the above-selected cases without this state, i.e. when the resonances $f_{0}(600), f_{0}(1500)$, and $f_{0}(1710)$ are represented respectively by the pole clusters of type (a), (g) and (b) in variant I (the "up" solution) and of type $\left(\mathbf{a}^{\prime}\right),\left(\mathbf{d}^{\prime}\right)$ and $\left(\mathbf{c}^{\prime}\right)$ in variant II. The $f_{0}(980)$ is represented by the poles on sheets II and III in both variants. In Table VIII we show a quality of description of each separate process for these cases in the frame of the best combined description of all three processes.

TABLE VIII: The quality of description of the data without the $f_{0}(1370)$.

\begin{tabular}{ccccc}
\hline \hline Variant & $\pi \pi$ scattering & $\pi \pi \rightarrow K \bar{K}$ & $\pi \pi \rightarrow \eta \eta, \eta \eta^{\prime}$ & The total \\
& $\chi^{2} /$ dof & $\chi^{2} /$ dof & $\chi^{2} /$ ndp & $\chi^{2} /$ dof \\
\hline I & $151.395 /(169-31) \approx 1.10$ & $155.056 /(120-29) \approx 1.70$ & 1.04 & $323.14 /(305-38) \approx 1.21$ \\
\hline II & $150.145 /(169-26) \approx 1.05$ & $160.056 /(120-26) \approx 1.70$ & 0.38 & $313.225 /(305-34) \approx 1.19$ \\
\hline \hline
\end{tabular}

When calculating $\chi^{2}$ in all cases, the following experimental points have been omitted as obviously strongly falling out from the energy dependence: from the $\pi \pi$ scattering data the points at $990 \mathrm{MeV}$ for the phase shift $\delta_{11}$ and for $\eta_{11}=\left|S_{11}\right|$, and for $\eta_{11}$ the point at $1650 \mathrm{MeV}$ as strongly violating the unitarity condition. From the $\pi \pi \rightarrow K \bar{K}$ data there were omitted the points at $1002,1208.9$ and $1235.7 \mathrm{MeV}$ for the $\eta_{12}=\left|S_{12}\right|$ and the points at 1073,1082 and $1387 \mathrm{MeV}$ as giving the anomalously big contribution to $\chi^{2}$. 

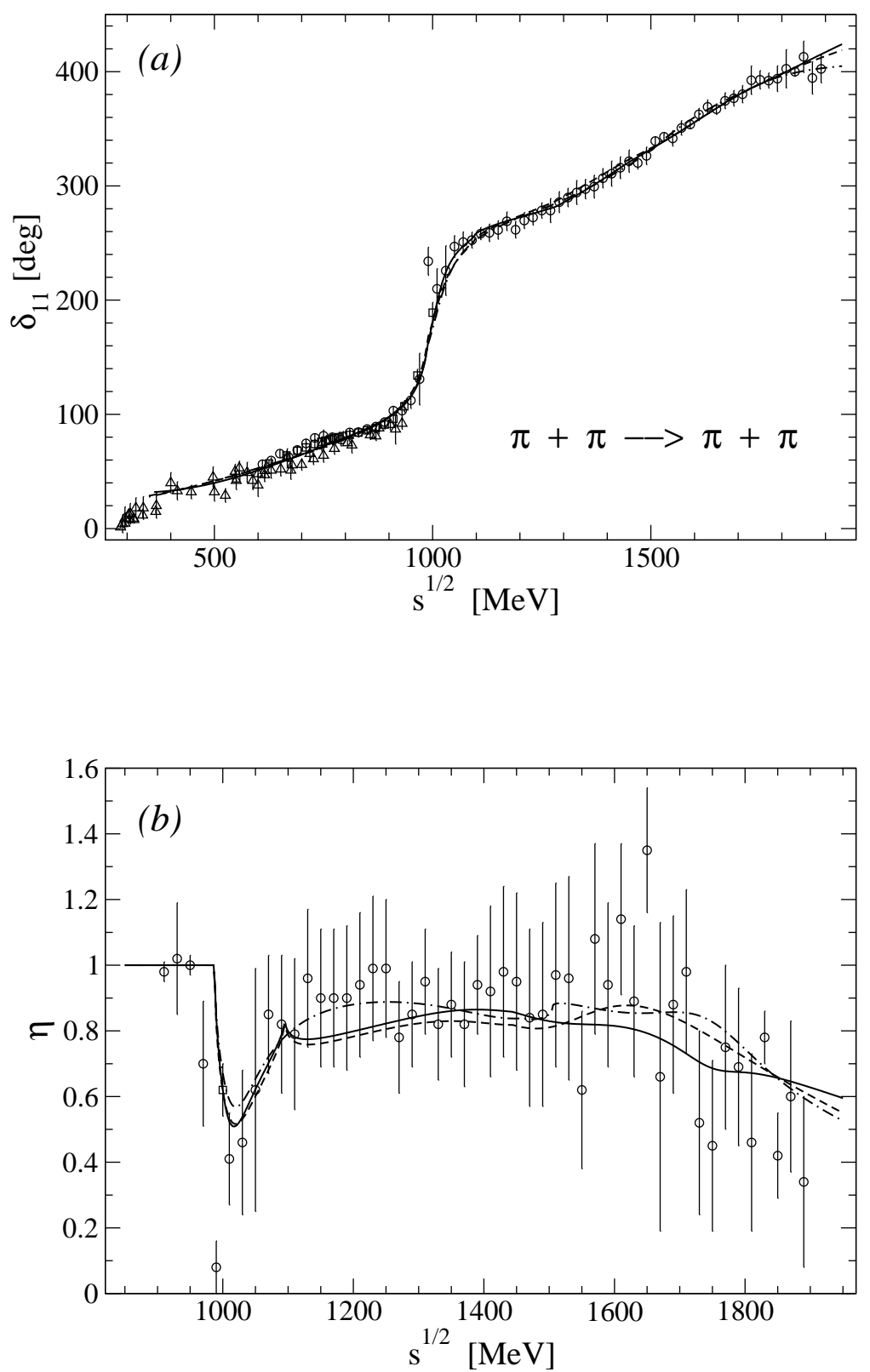

FIG. 3: The phase shift and modulus of the $S$-matrix element in the $S$-wave $\pi \pi$-scattering. The solid and short-dashed curves correspond to variant I, the "up" and "down" solutions, respectively; dash-dotted to variant II. The data are from Refs. 71 74].

One can see that the description of the $\pi \pi$ scattering without the $f_{0}(1370)$ is a bit improved whereas the one of the $\pi \pi \rightarrow K \bar{K}$ process is made slightly worse, especially as to the phase shift. Generally, an existence of the $f_{0}(1370)$ is for now a standard point of view. One ought to take into account also arguments to its favor in Ref. [31] (see Introduction). In any case, the existence of the $f_{0}(1370)$ does not contradict the considered data.

Let us make some more remarks. First, the fact that in variant II we obtain a better description than in variant I points to the importance of taking into account the $\eta \eta^{\prime}$ threshold explicitly. However, in variant II we encounter elements of some pseudo-background: these are the negative values of the $b$ coefficients related to an inelastic part of the background. The increasing inelastic part of the background implies a necessity to consider explicitly some physical 

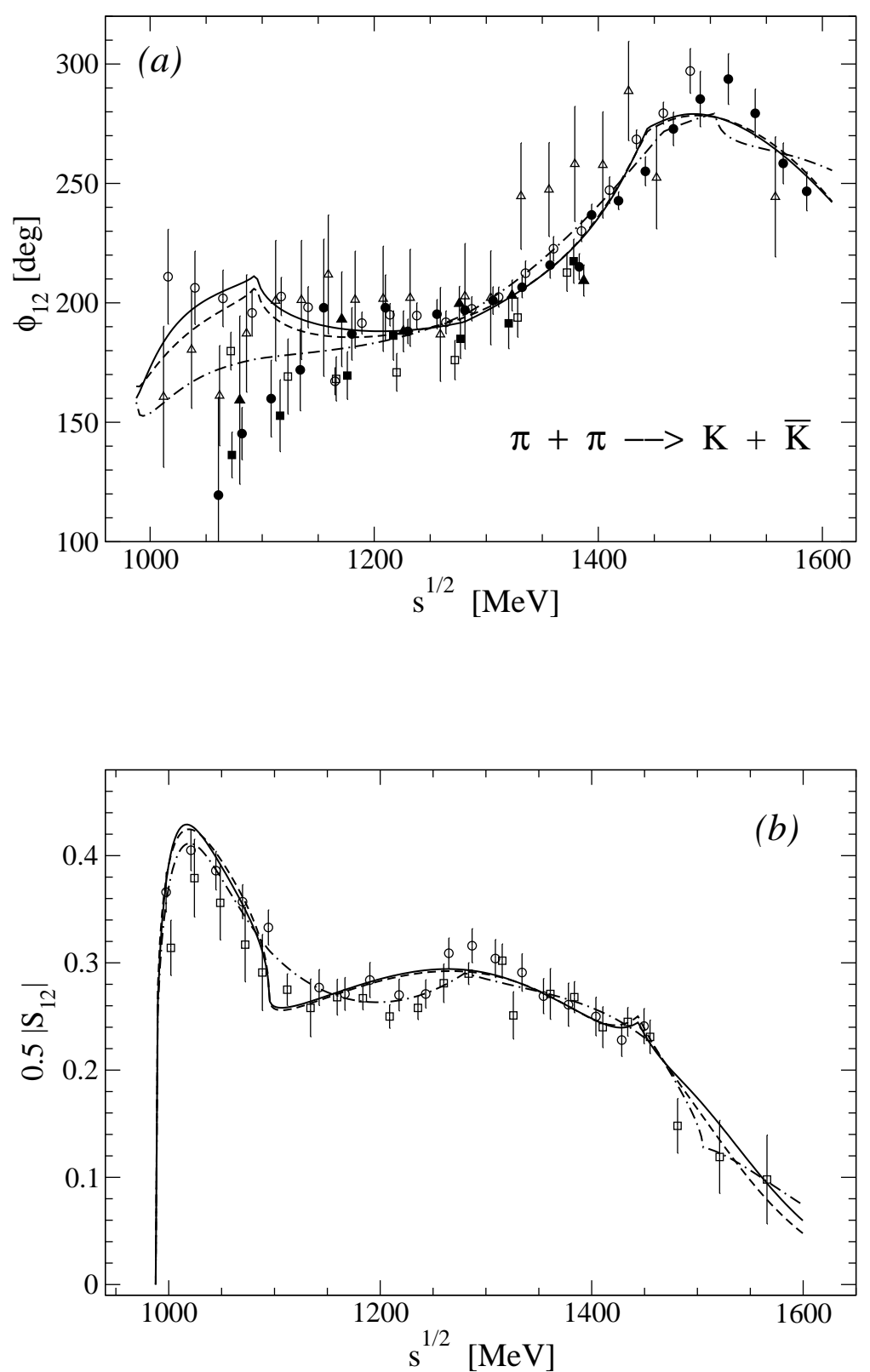

FIG. 4: The phase shift and modulus of the $S$-matrix element in $S$-wave of $\pi \pi \rightarrow K \bar{K}$. The solid and short-dashed curves correspond to variant I, the "up" and "down" solutions, respectively; dash-dotted to variant II. The data are from Ref. [75].

phenomenon, e.g., additional resonances or representation of resonances by other pole-clusters or the consideration in the uniformizing variable of other channel thresholds. The latter situation is the case here: the negative sign of the quantity $b_{2 \eta}^{\prime}=-0.7478$ implies the necessity of an explicit consideration of the $\eta \eta$-threshold branch-point. Therefore, as to the resonances below $1500 \mathrm{MeV}$ the more adequate description is variant I whereas for the ones above $1500 \mathrm{MeV}$ variant II.

It turns out that the state $f_{0}(980)$ lies slightly above the $K \bar{K}$ threshold. It is described by the pole on sheet II and by the shifted pole on sheet III under the $\eta \eta$ threshold without the corresponding poles on sheets VI and VII, as it was expected for standard clusters. This may suggest that the $f_{0}(980)$ is not the $q \bar{q}$ state and can be interpreted, 

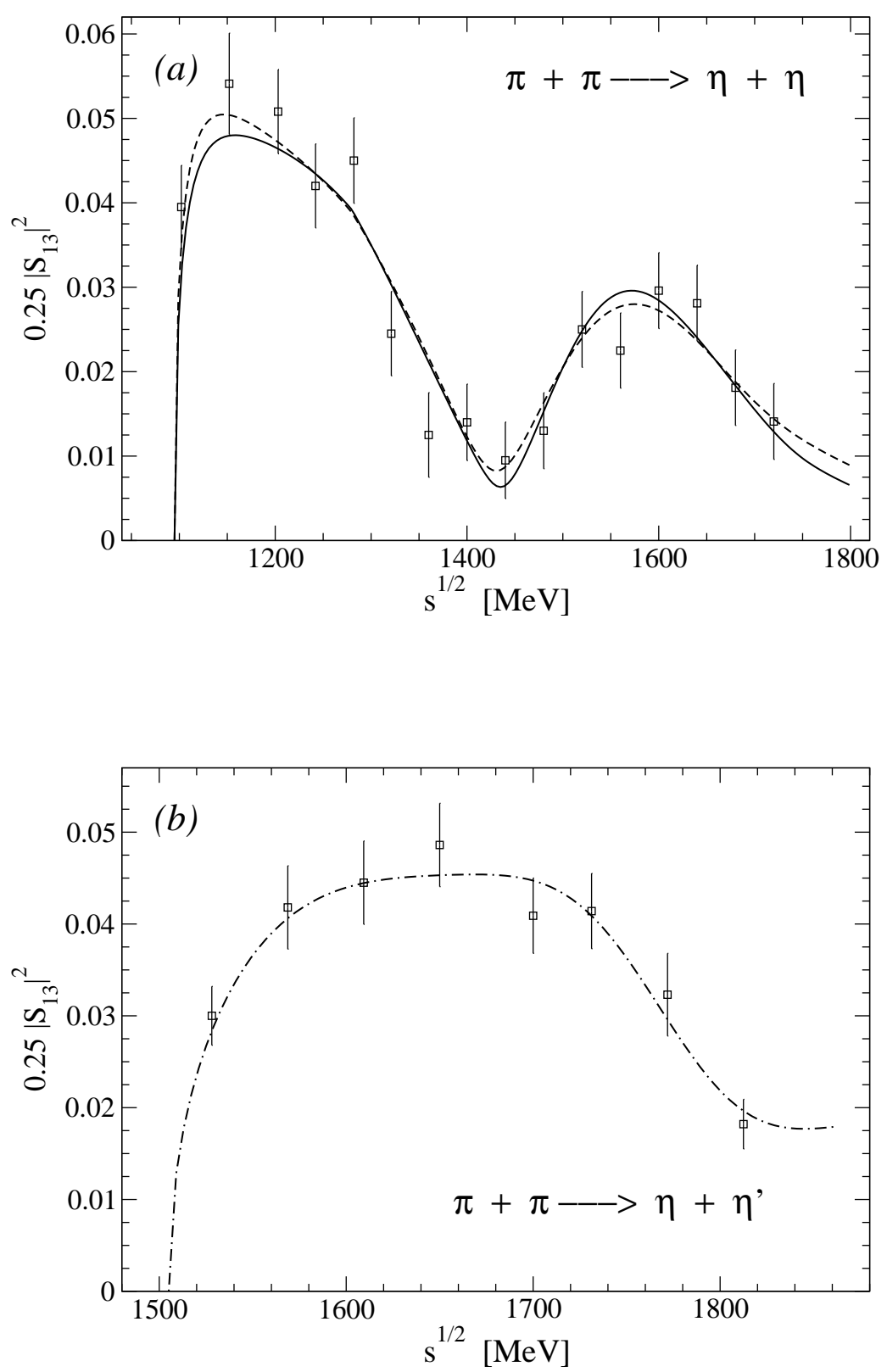

FIG. 5: The squared modules of the $\pi \pi \rightarrow \eta \eta$ (upper figure) and $\pi \pi \rightarrow \eta \eta^{\prime}$ (lower figure) $S$-wave matrix elements. The data are from Ref. [76] (upper figure) and from Ref. [77] (lower figure).

e.g., as a $\eta \eta$ bound state in accordance with the test discussed in Sec.II: the necessary condition for this is fulfilled. See, however, the further discussion of this matter in the last section.

As to a representation of the $f_{0}(600)$ and $f_{0}(980)$ states, both variants completely agree. The $f_{0}(1370)$ is described by the clusters of type (b) or (c) in various scenarios of variant I and of type $\left(\mathbf{b}^{\prime}\right)$ in variant II; this is reasonable taking into account the quark contents of the $K \bar{K}$ and $\eta \eta$ systems and the nearness of corresponding thresholds. From this we, therefore, deduce that a $s \bar{s}$ component of the $f_{0}(1370)$ is dominant. This interpretation quite explains why one did not find evidence for the existence of the $f_{0}(1370)$ [29] considering only the $\pi \pi$ scattering.

The $f_{0}(1500)$ is described by the cluster of type $(\mathbf{g})$ in variant I and of type $\left(\mathbf{d}^{\prime}\right)$ in variant II. The former indicates 
approximately equal coupling constants of this state to the $\pi \pi, K \bar{K}$ and $\eta \eta$ systems, which apparently could point up to its glueball nature. The latter tells on the approximately equal coupling of this state with the $\pi \pi$ and $K \bar{K}$ channels whereas the coupling with the $\eta \eta^{\prime}$ channel is suppressed; these facts also point up to its glueball nature [16]. Therefore, we deduce a dominant glueball component of the $f_{0}(1500)$.

Finally, the $f_{0}(1710)$ is described by the clusters of type (b) or (c) in various scenarios of variant I and of type $\left(\mathbf{c}^{\prime}\right)$ in variant II. Taking also into account the quark contents of the $\eta \eta^{\prime}$ system this could point to the dominant $s \bar{s}$ component of this state.

All these conclusions agree quite well with the previous model-independent 2- and 3-channel analyses 25-27, 30 , 3740] where other uniformizing variables were used.

\section{DISCUSSION AND CONCLUSIONS}

The combined analysis of data on the $\pi \pi \rightarrow \pi \pi, K \bar{K}, \eta \eta, \eta \eta^{\prime}$ processes in the channel with $I^{G} J^{P C}=0^{+} 0^{++}$is carried out in the framework of the model-independent approach that is based on analyticity and unitarity and uses an uniformization procedure. A new uniformizing variable was used in which, in additional to the right-hand branchpoints related with the thresholds of the analyzed channels, there is taken into account the left-hand branch-point at $s=0$ related to the $\pi \pi$ scattering in the crossed channels.

In the analysis of the processes $\pi \pi \rightarrow \pi \pi, K \bar{K}, \eta \eta$ it is shown that the data admit two possibilities for parameters of the $f_{0}(600)$ with mass, relatively near to the $\rho$-meson mass, and with total width about 640 and $1000 \mathrm{MeV}$. These two possibilities are related to two found solutions, admissible by the data below $1 \mathrm{GeV}$ for the phase shift of the $\pi \pi$ scattering amplitude: "up" and "down". As to the combined description of the considered processes it is impossible to prefer any of these solutions. However, the "up" solution remarkably accord with prediction by Weinberg [6] with respect to the mass and the width, the "down" one to the mass. These values of mass and width, calculated with help of formula (6) from the pole position on sheet II, correspond to most of the Breit-Wigner values of Refs. [79] (analysis of several processes with pseudoscalar mesons) and [80] (GAMS Collaboration, analysis of the reaction $p p \rightarrow p p \pi^{0} \pi^{0}$ ).

Furthermore, we have considered all relevant possibilities of representation of resonances by pole clusters (the 3channel resonances are represented by seven types of the pole clusters). It is shown that for the "up" solution there are four scenarios of representation of resonances $f_{0}(1370), f_{0}(1500)$ (as the superposition of two states, broad and narrow) and $f_{0}(1710)\left(f_{0}(600)\right.$ and $f_{0}(980)$ are given by the pole clusters of the same types in all cases) giving about the similar description of the above processes and, however, the quite different parameters of some resonances. For the $f_{0}(600), f_{0}(1370)$ and $f_{0}(1710)$ a spread of values is obtained for the masses and widths $605-735$ and $567-686 \mathrm{MeV}$, 1326-1404 and 223-345 MeV, and 1751-1759 and 118-207 MeV, respectively. On the other hand, the results for the $f_{0}(980)$ and $f_{0}(1500)$ are more stable and confirm conclusions of our previous analyses 25-27, 30, 37-39].

Note a quite stable result for the mass and width with rather small errors for the $f_{0}(980): m_{r e s} \approx 1005-1008 \mathrm{MeV}$, $\Gamma_{\text {tot }} \approx 45-54 \mathrm{MeV}$. Arrangement of the poles and zeroes on the Riemann surface, which describe this state, may suggest that the $f_{0}(980)$ is not the $q \bar{q}$ state and can be interpreted, e.g., as a $\eta \eta$ bound state; in any case the necessary condition for this is fulfilled. However, following the listings PDG 1], the mass of this state is obtained above the $K \bar{K}$ threshold in analyses of $\pi \pi$ scattering, of multi-channel $\pi \pi$ scattering $\left(\pi \pi \rightarrow \pi \pi, K \bar{K}, \eta \eta, \eta \eta^{\prime}\right)$ and of processes $\bar{p} p(n) \rightarrow M_{1} M_{2} M_{3}$, whereas below the $K \bar{K}$ threshold in analyses of the decays of $D^{+}-, B^{+}-, J / \psi_{-}$, and $Z-$ bosons, of processes $e^{+} e^{-} \rightarrow M_{1} M_{2} \gamma, \phi M_{1} M_{2} \gamma, e^{+} e^{-} M_{1} M_{2}, M_{1} M_{2} X$, and of $p p \rightarrow p p M_{1} M_{2}$. Since the mass value below the $K \bar{K}$ threshold is important for a dynamical interpretation of the $f_{0}(980)$ as a $K \bar{K}$ molecule [22 24] it seems that the nature of this state is more complicated than a simple $\eta \eta$ bound state or $K \bar{K}$ molecule. From the point of view of the quark structure these two possibilities are the 4-quark states. It seems this is consistent somehow with arguments in favor of the 4-quark nature of $f_{0}(980)$ in work of [19].

In view of prolonging discussions of a question, whether the $f_{0}(1370)$ exists or not (see the discussion of this matter in Introduction), we considered a description of the multi-channel $\pi \pi$ scattering without this state. We concluded that an existence of the $f_{0}(1370)$ does not contradict the considered data. The description of the $\pi \pi$ scattering is a bit improved whereas the one of the $\pi \pi \rightarrow K \bar{K}$ process is made worse, especially as to the phase shift.

The $f_{0}(1370)$ (if it exists) and $f_{0}(1710)$ have a dominant $s \bar{s}$ component. Conclusion about the $f_{0}(1370)$ agrees quite well with the conclusion drawn by the Crystal Barrel Collaboration [81] where the $f_{0}(1370)$ is identified as $\eta \eta$ resonance in the $\pi^{0} \eta \eta$ final state of the $\bar{p} p$ annihilation at rest. Interpretation of the $f_{0}(1370)$ as dominated by the $s \bar{s}$ component explains also quite well why one did not find this state considering only the $\pi \pi$ scattering. Conclusion about the $f_{0}(1710)$ is quite consistent with the experimental facts that this state is observed in $\gamma \gamma \rightarrow K_{S} \bar{K}_{S}$ [82] but not observed in $\gamma \gamma \rightarrow \pi^{+} \pi^{-}$[83].

As to the $f_{0}(1500)\left(m_{r e s}=1510 \mathrm{MeV}, \Gamma_{t o t}=397 \mathrm{MeV}\right)$ we suppose that it is the eighth component of octet mixed with the glueball being dominant in this state. Its largest width among the enclosing states points also to its glueball nature [84]. Note that in the PDG tables on the $f_{0}(1500)$ listing, an average value for the width of $109 \pm 7 \mathrm{MeV}$ 
is cited. However, there one indicates only the results of analyses of meson production processes, and in the few cases where the results of combined analyses of coupled processes are cited, authors did not use the representations of the multi-channel resonances by pole clusters (this is especially important in the case of wide resonances), i.e., they did not apply all aspects of the multi-channel analysis. On the other hand, one can see from the data on scattering processes, analyzed here [71], that the energy dependence of observed quantities do not demonstrate a pronounced structure in the $1500 \mathrm{MeV}$ region, which is needed for the narrow resonance. Therefore, it is reasonable to suggest that in this region there is a superposition of two states, a wide and a narrow one.

It is known that there is a number of properties of the scalar mesons which do not allow for a satisfactory setup the lowest nonet. The main observations are the approximate equal masses of $f_{0}(980)$ and $a_{0}(980)$ and the $s \bar{s}$ dominance in the wave function of the $f_{0}(980)$. If these states are in the same nonet then the $f_{0}(980)$ must be heavier than $a_{0}(980)$ by about $250-300 \mathrm{MeV}$ due to the mass difference of $s$ - and $u$-quark. Exclusion of the $f_{0}(980)$ as a non $q \bar{q}$ state and discovery of the $K_{0}^{*}$-doublet (if it will be confirmed) moves off a number of these problems.

One can propose the following assignment of scalar mesons lying below $1.9 \mathrm{GeV}$ to lower nonets [27]. The lowest nonet: the isovector $a_{0}(980)$, the isodoublet $K_{0}^{*}(900)$, and $f_{0}(600)$ and $f_{0}(1370)$ as mixtures of the 8th component of octet and the $\mathrm{SU}(3)$ singlet. Then the Gell-Mann-Okubo (GMO) formula of

$$
3 m_{f_{8}}^{2}=4 m_{K_{0}^{*}}^{2}-m_{a_{0}}^{2}
$$

gives $m_{f_{8}}=910 \mathrm{MeV}$. For this nonet is seems to be important to test the nature of strange scalar meson $K_{0}^{*}(900)$ in a model-independent way. This will be the subject of a forthcoming paper [68].

In the relation for the masses of the nonet

$$
m_{\sigma}+m_{f_{0}(1370)}=2 m_{K_{0}^{*}}
$$

the left-hand side is by about $18 \%$ larger than the right-hand one.

For the next nonet of the radial excitations we find: $a_{0}(1450), K_{0}^{*}(1450)$, and $f_{0}(1500)$ and $f_{0}(1710)$, the $f_{0}(1500)$ being mixed with a glueball which is dominant in this state. From the GMO formula we set $m_{f_{8}} \approx 1453 \mathrm{MeV}$. In the formula

$$
m_{f_{0}(1500)}+m_{f_{0}(1710)}=2 m_{K_{0}^{*}(1450)}
$$

the left-hand side is by about $12.5 \%$ larger than the right-hand one.

This assignment removes a number of prior questions and does not rise new ones. The mass formulas indicate to a non-trivial mixing scheme. Breaking of the relations (13) and (14) tells us that the $\sigma-f_{0}(1370)$ and $f_{0}(1500)-f_{0}(1710)$ systems get additional contributions absent in the $K_{0}^{*}(900)$ and $K_{0}^{*}(1450)$, respectively. A search of the adequate mixing scheme is complicated by the circumstance that here there is also a remainder of chiral symmetry, though, on the other hand, this permits one to predict correctly, e.g., the $\sigma$-meson mass.

\section{Acknowledgments}

The authors thank Thomas Gutsche and Mikhail Ivanov for useful discussions. This work was supported in part by the Heisenberg-Landau Program, the RFBR grant 10-02-00368-a, the Votruba-Blokhintsev Program for Cooperation of the Czech Republic with JINR (Dubna), the Grant Agency of the Czech Republic (Grant No.202/08/0984) and by Federal Targeted Program "Scientific and scientific-pedagogical personnel of innovative Russia" Contract No. 02.740.11.0238.

\section{Appendix A: Analytic continuation of the 3-channel $S$-matrix elements to unphysical sheets}

Here we show, for convenience, formulas of the analytic continuations of the 3 -channel $S$-matrix elements to unphysical sheets of the Riemann surface in terms of those on sheet I (the physical sheet) - $S_{\alpha \beta}^{I}$ that have only zeros (beyond the real axis) corresponding to resonances, at least, around the physical region. In Ref. [40] the general formula was given for the case of $\mathrm{N}$ channels and as example for three channels. The direct derivation of these formulas requires rather bulky algebra. It can be simplified if we use a circumstance that the $K$-matrix has the same value in all sheet of the Riemann surface of the $S$-matrix. This fact follows from Hermiticity of the $K$-matrix $K=K^{+}$which means that the $K$-matrix does not try discontinuity when going across unitarity cuts. Then after some algebra, one can obtain formulas under interest shown below in the table. In Table IX] the superscript $I$ is omitted to simplify 
TABLE IX: Analytic continuations of the 3-channel $S$-matrix elements to unphysical sheets

\begin{tabular}{ccccccccc}
\hline \hline Process & I & II & III & IV & V & VI & VII & VIII \\
\hline $1 \rightarrow 1$ & $S_{11}$ & $1 / S_{11}$ & $S_{22} / D_{33}$ & $D_{33} / S_{22}$ & $\operatorname{det} S / D_{11}$ & $D_{11} / \operatorname{det} S$ & $S_{33} / D_{22}$ & $D_{22} / S_{33}$ \\
$1 \rightarrow 2$ & $S_{12}$ & $i S_{12} / S_{11}$ & $-S_{12} / D_{33}$ & $i S_{12} / S_{22}$ & $i D_{12} / D_{11}$ & $-D_{12} / \operatorname{det} S$ & $i D_{12} / D_{22}$ & $D_{12} / S_{33}$ \\
$2 \rightarrow 2$ & $S_{22}$ & $D_{33} / S_{11}$ & $S_{11} / D_{33}$ & $1 / S_{22}$ & $S_{33} / D_{11}$ & $D_{22} / \operatorname{det} S$ & $\operatorname{det} S / D_{22}$ & $D_{11} / S_{33}$ \\
$1 \rightarrow 3$ & $S_{13}$ & $i S_{13} / S_{11}$ & $-i D_{13} / D_{33}$ & $-D_{13} / S_{22}$ & $-i D_{13} / D_{11}$ & $D_{13} / \operatorname{det} S$ & $-S_{13} / D_{22}$ & $i S_{13} / S_{33}$ \\
$2 \rightarrow 3$ & $S_{23}$ & $D_{23} / S_{11}$ & $i D_{23} / D_{33}$ & $i S_{23} / S_{22}$ & $-S_{23} / D_{11}$ & $-D_{23} / \operatorname{det} S$ & $i D_{23} / D_{22}$ & $i S_{23} / S_{33}$ \\
$3 \rightarrow 3$ & $S_{33}$ & $D_{22} / S_{11}$ & $\operatorname{det} S / D_{33}$ & $D_{11} / S_{22}$ & $S_{22} / D_{11}$ & $D_{33} / \operatorname{det} S$ & $S_{11} / D_{22}$ & $1 / S_{33}$ \\
\hline \hline
\end{tabular}

the notation, $\operatorname{det} S$ is the determinant of the $3 \times 3 S$-matrix on sheet I, $D_{\alpha \beta}$ is the minor of the element $S_{\alpha \beta}$, that is, $D_{11}=S_{22} S_{33}-S_{23}^{2}, D_{22}=S_{11} S_{33}-S_{13}^{2}, D_{33}=S_{11} S_{22}-S_{12}^{2}, D_{12}=S_{12} S_{33}-S_{13} S_{23}, D_{23}=S_{11} S_{23}-S_{12} S_{13}$, etc.

[1] K. Nakamura et al. (PDG), J. Phys. G 37, 075021 (2010).

[2] C. Amsler and N.A. Törnqvist, Phys. Reports 389, 61 (2004).

[3] D.V. Bugg, , Phys. Reports 397, 257 (2004).

[4] F.E. Close and N.A. Törnqvist, J. Phys. G 28, R249 (2002).

[5] E. Klempt and A. Zaitsev, Phys. Reports 454, 1 (2007).

[6] S. Weinberg, Phys. Rev. Lett. 65, 1177 (1990).

[7] R. Kamiński, G. Mennessier, and S. Narison, Phys. Lett. B 680, 148 (2009); arXiv: 0904.2555 [hep-ph].

[8] C. McNeile, Nucl. Phys. B (Proc. Suppl.) 186, 264 (2009).

[9] Y. Chen et al., Phys. Rev. D 73, 014516 (2006).

[10] Yu. Troyan et al., JINR Rapid Communications 5-91, 33 (1998).

[11] N.N. Achasov and G.N. Shestakov, Phys. Rev. D 49, 5779 (1994).

[12] F.J. Gilman and H. Harari, Phys. Rev. 165, 1803 (1968).

[13] J. Ellis and J. Lánik, Phys. Lett. B 150, 289 (1985).

[14] V.V. Anisovich, Physics-Uspekhi 41, 419 (1998); V.V. Anisovich, D.V. Bugg, and A.V. Sarantsev, Phys. Rev. D 58, 111503 (1998).

[15] F.Giacosa, T. Gutsche, V. E. Lyubovitskij, and A. Faessler, Phys. Rev. D 72, 094006 (2005).

[16] C. Amsler and F.E. Close, Phys. Rev. D 53, 295 (1996).

[17] D. Morgan, Phys. Lett. B 51, 71 (1974).

[18] N.A. Törnqvist, Phys. Rev. Lett. 49, 624 (1982).

[19] N.N. Achasov, Nucl. Phys. A 675, 279c (2000).

[20] J. Lánik, Phys. Lett. B 306, 139 (1993).

[21] N.A. Törnquist and M. Roos, Phys. Rev. Lett. 76, 1575 (1996).

[22] J. Weinstein and N. Isgur, Phys. Rev. Lett. 48, 659 (1982); Phys. Rev. D 27, 588 (1983); 41, 2236 (1990).

[23] G. Jansen, B.C. Pearce, K. Holinde, and J. Speth, Phys. Rev. D 52, 2690 (1995).

[24] T. Branz, T. Gutsche, and V. E. Lyubovitskij, Eur. Phys. J. A 37, 303 (2008).

[25] Yu.S. Surovtsev and R. Kamiński, Frascati Physics Series XLVI, 669 (2007).

[26] Yu.S. Surovtsev, P. Bydžovský, R. Kamiński, and M. Nagy, Int. J. Mod. Phys. A 24, 586 (2009).

[27] Yu.S. Surovtsev, P. Bydžovský, R. Kamiński, and M. Nagy, Phys. Rev. D 81, 016001 (2010).

[28] P. Minkowski and W. Ochs, Eur. Phys. J. C 9, 283 (1999); arXiv: hep-ph/0209223; hep-ph/0209225.

[29] W. Ochs, AIP Conf. Proc. 1257, 252 (2010) arXiv:1001.4486 [hep-ph]].

[30] Yu.S. Surovtsev, D. Krupa, and M. Nagy, Eur. Phys. J. A 15, 409 (2002).

[31] D.V. Bugg, Eur. Phys. J. C 52, 55 (2007); arXiv: hep-ex/0706.1341; arXiv: 0710.4452 [hep-ex].

[32] C. Amsler et al., Phys. Lett. B 291, 347 (1992).

[33] A. Abele et al., Nucl. Phys. A 609, 562 (1996).

[34] M. Ablikim et al., Phys. Lett. B 607, 243 (2005).

[35] D. Alde et al., Eur. Phys. J. A 3, 361 (1998).

[36] R. Molina, D. Nicmorus, and E. Oset, Phys. Rev. D 78, 114018 (2008).

[37] Yu.S. Surovtsev, D. Krupa, and M. Nagy, Phys. Rev. D 63, 054024 (2001).

[38] Yu.S. Surovtsev, D. Krupa, and M. Nagy, AIP Conf. Proc. 17, 357 (2004).

[39] Yu.S. Surovtsev, D. Krupa, and M. Nagy, Czech. J. Phys. 56, 807 (2006).

[40] D. Krupa, V.A. Meshcheryakov, and Yu.S. Surovtsev, Nuovo Cim. A 109, 281 (1996).

[41] V.V. Anisovich et al., Phys. Atom. Nucl. 60, 1410 (2000); V.V. Anisovich, V.A. Nikonov, and A.V. Sarantsev, Phys. Atom. 
Nucl. 65, 1545 (2002).

[42] D. Alde et al., Zeit. Phys. C 66, 375 (1995); Yu. D. Prokoshkin et al., Physics-Doklady 342, 473 (1995); F. Binon et al., Nuovo Cim. A 78, 313 (1983), 80, 363 (1984).

[43] S.J. Lindenbaum and R.S. Longacre, Phys. Lett. B 274, 492 (1992); A. Etkin et al., Phys. Rev. D 25, 1786 (1982).

[44] V.V. Anisovich et al., Phys. Lett. B 323, 233 (1994).

[45] C.Amsler et al., Phys. Lett. B 342, 433 (1995).

[46] F.E. Close and A. Kirk, Eur. Phys. J. C 21, 531 (2001).

[47] S. Narison, Nucl. Phys. B 509, 312 (1998).

[48] A.V. Anisovich, V.V. Anisovich, and A.V. Sarantsev, Phys. Rev. D 62, 051502 (2000).

[49] W. Lee and D. Weingarten, Phys. Rev. D 61, 014015 (2000).

[50] M. Chanowitz, Phys. Rev. Lett. 95, 172001 (2005).

[51] M. Albaladejo and J.A. Oller, Phys. Rev. Lett. 101, 252002 (2008).

[52] D.V. Bugg, Phys. Lett. B 632, 471 (2006).

[53] M. Ablikim et al., Phys. Lett. B 633, 681 (2006).

[54] S. Descotes-Genon and B. Moussallam, Eur. Phys. J. C 48, 553 (2006).

[55] Z.Y. Zhou and H.Q. Zheng, Nucl. Phys. A 775, 212 (2006).

[56] C. Cawlfield et al., Phys. Rev. D 74, 031108R (2006).

[57] J.M. Link et al., Phys. Lett. B 653, 1 (2007).

[58] Yu.S. Surovtsev, T. Branz, T. Gutsche, and V.E. Lyubovitskij, Physics of Particles and Nuclei 41, 990 (2010).

[59] B. Aubert et al., Phys. Rev. D 76, 011102R (2007).

[60] S.N. Cherry and M.R. Pennington, Nucl. Phys. A 688, 823 (2001).

[61] S. Kopp et al., Phys. Rev. D 63, 092001 (2001).

[62] J.M. Link et al., Phys. Lett. B 535, 43 (2002); 621, 72 (2005).

[63] S. Godfrey and N. Isgur, Phys. Rev. D 32, 189 (1985).

[64] N.A. Törnqvist, arXiv: hep-ph/0204215.

[65] V.V. Anisovich, Yu.D. Prokoshkin, and A.V. Sarantsev, Nucl. Phys. Proc. Suppl. A 56, 270 (1997).

[66] F.E. Close and N.A. Törnqvist, J. Phys. G 28, R249 (2002).

[67] M.K. Volkov and V.L. Yudichev, Yad. Fiz. 65, 1701 (2002).

[68] Yu. S. Surovtsev, P. Bydžovský, and V. E. Lyubovitskij, in preparation.

[69] D. Morgan and M.R. Pennington, Phys. Rev. D 48, 1185 (1993).

[70] K.J. Le Couteur, Proc. R. London, Ser. A 256, 115 (1960); R.G. Newton, J. Math. Phys. 2, 188 (1961); M. Kato, Ann. Phys. 31, 130 (1965).

[71] B. Hyams et al., Nucl. Phys. B 64, 134 (1973); 100, 205 (1975).

[72] A. Zylbersztejn et al., Phys. Lett. B 38, 457 (1972); P. Sonderegger, P. Bonamy, in Proc. 5th Int. Conference on Elementary Particles, Lund, 1969, 372; J.R. Bensinger et al., Phys. Lett. B 36, 134 (1971); J.P. Baton et al., Phys. Lett. B 33, 525 (1970); 33, 528 (1970); P. Baillon et al., Phys. Lett. B 38, 555 (1972); L. Rosselet et al., Phys. Rev. D 15, 574 (1977); A.A. Kartamyshev et al., Pis'ma Zh. Eksp. Theor. Fiz. 25, 68 (1977); A.A. Bel'kov et al., Pis'ma Zh. Eksp. Theor. Fiz. 29, 652 (1979).

[73] S.D. Protopopescu et al., Phys. Rev. D 7, 1279 (1973).

[74] P. Estabrooks and A.D. Martin, Nucl. Phys. B 79, 301 (1974).

[75] W. Wetzel et al., Nucl. Phys. B 115, 208 (1976); V.A. Polychronakos et al., Phys. Rev. D 19, 1317 (1979); P. Estabrooks, Phys. Rev. D 19, 2678 (1979); D. Cohen et al., Phys. Rev. D 22, 2595 (1980); G. Costa et al., Nucl. Phys. B 175, 402 (1980); A. Etkin et al., Phys. Rev. D 25, 1786 (1982).

[76] F. Binon et al., Nuovo Cimento A 78, 313 (1983).

[77] F. Binon et al., Nuovo Cimento A 80, 363 (1984).

[78] R. Kamiński et al., Z. Phys. C 74, 79 (1997).

[79] N.A. Törnqvist and M. Roos, Phys. Rev. Lett. 76, 1575 (1996).

[80] D.M. Alde et al., Phys. Lett. B 397, 350 (1997).

[81] C. Amsler et al., Phys. Lett. B355, 425 (1995).

[82] S. Braccini, Frascati Phys. Series XV, 53 (1999).

[83] R. Barate et al., Phys. Lett. B 472, 189 (2000).

[84] V.V. Anisovich et al., Nucl. Phys. A (Proc. Suppl.) 56, 270 (1997). 